\title{
DEFORMACIÓN INTENCIONAL DEL CRÁNEO EN POBLACIONES ARQUEOLÓGICAS DE ARICA, CHILE: ANÁLISIS PRELIMINAR DE MORFOMETRÍA GEOMÉTRICA CON USO DE RADIOGRAFÍAS CRANEOFACIALES
}

\author{
INTENTIONAL CRANIAL DEFORMATION IN ARCHAEOLOGICAL \\ POPULATIONS OF ARICA (CHILE): PRELIMINARY GEOMETRIC \\ MORPHOMETRICS ANALYSIS USING CRANIOFACIAL RADIOGRAPHS
}

\author{
Germán Manríquez ${ }^{1}$, Fermín E. González-Bergás ${ }^{2}$, \\ Juan Carlos Salinas ${ }^{3}$ y Oscar Espoueys ${ }^{4}$
}

\begin{abstract}
La deformación intencional craneana tiene como consecuencia la alteración permanente de los atributos anatómicos externos del cráneo humano. Entre las poblaciones prehispánicas del extremo norte de Chile esta práctica se extendió por un lapso de cinco mil años. En algunos casos, la presencia en el cráneo de restos bioculturales impide la aplicación de las técnicas morfométricas normalmente utilizadas en cráneo seco. Con el fin de superar esta limitación y conocer el patrón de variación de la forma craneana de muestras representativas de las poblaciones prehispánicas de Arica, se obtuvieron radiografías laterales que fueron analizadas mediante morfometría geométrica. Los resultados del presente trabajo muestran que: (1) la variación de los componentes de la forma craneana depende de la magnitud y dirección con que se aplicaron y orientaron originalmente los aparatos deformadores sobre los ejes anatómicos mayores del cráneo, (2) la deformación intencional involucra a los hitos anatómicos del neurocráneo y de la cara y (3) el tipo de la deformación intencional sería independiente del período cronológico durante el cual se llevó a cabo esta práctica, sugiriendo la ocurrencia de una continuidad morfológica entre las poblaciones arqueológicas de la costa y valles de Arica. Se discuten las consecuencias de esta discrepancia con los resultados obtenidos al aplicar morfometría convencional, se constata la importancia del uso de las técnicas radiográficas y la morfometría geométrica para comprender las consecuencias morfológicas de la deformación intencional del cráneo, y se discuten las eventuales aplicaciones clínicas de este enfoque morfométrico en poblaciones actuales.
\end{abstract}

Palabras claves: deformación intencional, Arica-Chile, morfometría geométrica, radiografía craneofacial.

Intentional cranial deformation is a long lasting and worldwide form of permanent modification of the main human head distinctive anatomical attributes. In northern Chile it was extensively done during five thousand years by populations which inhabited the Coast and Valleys from Arica. In spite of the importance that the study of this practice has had in anthropological research, biocultural remains such as hear, mould and textils preclude the morphometric analyses commonly performed onto the clean skull. In order to avoid these limitations and to know the pattern of skull shape variation, geometric morphometric methods and craneofacial radiographic technics were applied on non deformed and intentionally deformed samples of Arica's archaeological populations. As a control sample radiographs obtained from modern Chileans were used. Radiographic and geometric morphometric evidences here presented show that: (1) variation of skull shape components of intentionally deformed crania is explained by both the force and the direction of the deforming devices originally applied, (2) intentional deformation affects anatomical landmarks located in the neurocrania as well as the face, (3) there would be a morphological continuity among populations representatives of Archaic and Agro-ceramic cultural traditions. The role of geometric morphometric and radiographic technics to asses the problem of intentional cranial deformation in archaeological and modern human populations as well as the discrepancy between our results and the results obtained by means of the traditional morphometric methods are discussed.

Key words: Intentional cranial deformation, Arica-Chile, geometric morphometrics, radiography.

\footnotetext{
1 Programa de Genética Humana, I.C.B.M., Facultad de Medicina y Departamento de Antropología, Facultad de Ciencias Sociales, Universidad de Chile, Casilla 70061, Santiago, Chile. gmanriqu@med.uchile.cl

2 Servicio Dento Máxilo-Facial, Hospital Clínico, Universidad de Chile, Santiago, Chile.

3 Departamento de Prótesis Estomatológica, Facultad de Odontología, Universidad de Chile, Santiago, Chile.

4 Museo Chileno de Arte Precolombino y Museo Nacional de Historia Natural, Santiago, Chile. oespoueys@yahoo.es
} 
La deformación intencional del cráneo consiste en el proceso de modificación del patrón de crecimiento y desarrollo craneanos mediante el uso en los primeros años de vida de aparatos deformadores utilizados de manera combinada o por separado, aplicados con distinta intensidad. Estos pueden ser rígidos, como tablillas, o flexibles, como fajas, cintillos, vendas y almohadillas, entre otros. Su principal consecuencia es la alteración en magnitud y dirección de los vectores que describen la forma del cráneo. La modificación intencional de la forma del cráneo se ha practicado en poblaciones humanas de muy distinto origen geográfico, períodos y culturas, desde el paleolítico superior hasta el siglo XX (Dembo e Imbelloni 1938; Gerszten y Gerszten 1995; Özbek 2001; Stewart 1943; Weiss 1961), habiéndose descrito, incluso, en restos fósiles de Homo neanderthalensis (Trinkaus 1982).

En muestras precolombinas de Norteamérica y Sudamérica se ha observado también, que como resultado de esta práctica ocurre un aumento en el número de huesos intercalares (Ossenberg 1970), y en la complejidad de las suturas del cráneo, estimada mediante el grado de interdigitación (Anton et al. 1992). Además, se observa una mayor frecuencia en el cierre temprano de la sutura sagital (White 1966) y un incremento de la vascularización endocraneal en la zona afectada por la deformación (O’Loughlin 1996). Dichos cambios se dan con más frecuencia en las regiones occipital y frontal del cráneo, que reciben la mayor impronta biomecánica de los aparatos deformadores. En el caso de los huesos intercalares, a pesar de que se ha sugerido la participación de un componente hereditario por encontrarse este rasgo presente en el estado fetal (El-Najjar y Dawson 1977), existe consenso en que su mayor frecuencia está asociada a la presión ejercida por la acción de aparatos deformadores aplicados intencionalmente sobre el cráneo (Anton et al. 1992; Benett 1965).

En el norte de Chile, esta práctica ocurrió por un lapso de, al menos, 5.000 años, en la región de Arica, y se aplicó tanto en grupos cazadores-recolectores marítimos de la Cultura Chinchorro, en el período Arcaico, como en grupos pescadores y agroalfareros de los períodos Temprano, Intermedio Temprano, Medio (Cultura Tiwanaku) e Intermedio Tardío o Desarrollos Regionales (Allison et al. 1981; Arriaza 2003; Aufderheide et al. 1993;
Bird 1943; Chacama 2004; Dauelsberg 1972; Espoueys et al. 1995a, 1995b; Llagostera 1989; Muñoz 1989, 2004; Romero 2004; Rothhammer et al. 1989; Schiappachasse et al. 1989; Standen 2003; Standen et al. 2004; Santoro et al. 2004; Uhle 1919), hasta su prohibición por la inquisición durante las campañas de extirpación de las idolatrías.

En los restos craneanos exhumados de los yacimientos arqueológicos de estas poblaciones es común observar la presencia de piel, cabello, máscaras de barro y turbantes (Dauelsberg 1972; SotoHeim 1987), existiendo amplio consenso respecto del rol que juegan las técnicas no invasivas en el análisis de este tipo de muestras (Hunt y Hopper 1996). Al respecto, en nuestro país se han empleado técnicas radiográficas para determinar el tipo de deformación craneana intencional en fardos funerarios (sitio Camarones 15, período Arcaico) (Soto-Heim 1987), y establecer la presencia de restos óseos del cráneo y postcráneo en estatuillas de momias (Playa Miller 8, período Arcaico) (Arriaza et al. 2002). También se ha diagnosticado en el postcráneo de restos humanos momificados, como huesos largos, vértebras, omóplatos y costillas, condiciones mórbidas asociadas con cuadros de neumonía, sarampión, influenza, varicela, treponematosis, hiperostosis, neumoconiosis silicosa y deficiencias nutricionales severas, las cuales habrían afectado a poblaciones arqueológicas que habitaron la costa y valles de Arica entre 7.800 a.p. (período Arcaico) y 600 a.p. (período Tardío) (Allison et al. 1982; Arriaza et al. 1984; Arriaza 1993; Aufderheide et al. 2002; Standen et al. 1984; Standen y Arriaza 2000). No obstante la enorme importancia antropológica de este material, y debido a que los remanentes bioculturales asociados al cráneo impiden el reconocimiento de la mayor parte de los hitos anatómicos sobre los que se basa el análisis cefalométrico, el uso de estas técnicas continúa ausente en los análisis antropométricos de los restos óseos arqueológicos provenientes de Arica.

Por otra parte, el conocimiento antropométrico de las poblaciones prehispánicas de Chile, en especial de los valles bajos y la costa del norte árido, se caracteriza por el amplio uso del análisis estadístico univariado y multivariado de mediciones (distancias entre hitos anatómicos) (Cocilovo 1975; Cocilovo et al. 1982; Cocilovo 1994; Rhode 2002; Rothhammer et al. 1982; Rothhammer y Silva 1990; Rothhammer et al. 2002; Vare- 
la y Cocilovo 2002). A pesar de representar un avance significativo respecto de la morfometría aplicada hasta mediados del siglo XX, su empleo presenta limitaciones inherentes al manejo de datos primarios lineales. Entre estos problemas destacan la falta de una adecuada partición de los componentes de la forma en relación a los componentes de tamaño, la ausencia de una definición inambigua de homología para los hitos anatómicos, la pérdida de poder estadístico debido a la sobreposición de las mediciones que cubren los ejes anatómicos mayores y la incapacidad de reproducir visualmente en dos o tres dimensiones el patrón de variación de la forma de los objetos biológicos (Bookstein 1991).

Estas dificultades estimularon el reciente desarrollo de la morfometría geométrica. Esta nueva herramienta del análisis biométrico es la síntesis de la biometría clásica y de las técnicas de transformación de grillas cartesianas de D'Arcy Thompson (1980[1917]) (Rohlf y Marcus 1993), y se caracteriza por (Bookstein 1991): (a) uso de hitos de morfocoordenadas y contornos como datos primarios con construcción de espacios multidimensionales de los componentes de la forma (espacios de Procusto y de Kendall), (b) remoción de las diferencias debidas a tamaño, rotación y traslación, y registro de la variación de la forma mediante sobreposición de hitos homólogos (análisis de Procusto), (c) obtención de un estimador del tamaño geométrico (tamaño del centroide), estadísticamente independiente de los estimadores de la forma (componentes de variación uniforme y variación no uniforme), (d) representación geométrica del patrón de variación de la forma (función de placa delgada) en morfoespacios de dos o tres dimensiones que recuperan la información espacial contenida en los objetos biológicos (análisis de relative warps), (e) puesta a prueba de hipótesis estadísticas y biológicas sobre la significación de las diferencias entre grupos respecto de la variación de los componentes de la forma y las variables independientes que, eventualmente, explican dichas diferencias (O'Higgins 2000). No obstante sus ventajas en comparación con los enfoques de la morfometría lineal, sólo recientemente esta herramienta se ha comenzado a aplicar al estudio de la variación de la forma del cráneo en poblaciones prehispánicas y protohistóricas de Chile (Manríquez et al. 2002, 2003, 2004; Manríquez y Llop 2004; Salinas et al. 2004).

\section{Objetivos, Hipótesis y Predicciones}

El objetivo de este estudio es mostrar las ventajas de aplicar las herramientas de la morfometría geométrica en cráneos deformados artificialmente, incluso aquellos que están cubiertos por restos bioculturales y, por lo mismo, no han sido utilizados hasta el presente en los análisis biométricos tradicionales. Dada la presencia de dichos restos, los análisis morfométricos se realizaron usando radiografías craneofaciales obtenidas según los procedimientos radiológicos estándar.

De acuerdo con nuestras hipótesis de nulidad, la práctica de la deformación intencional del cráneo no altera significativamente el patrón de variación morfológica del cráneo (H01), y es semejante entre distintos períodos cronológicos (H02). Si estas hipótesis son ciertas, entonces se espera no encontrar diferencias significativas entre cráneos no deformados y deformados intencionalmente (predicción derivada de H01), ni entre los cráneos no deformados o entre los cráneos deformados intencionalmente que pertenecen a distintos tipos y/o períodos cronológicos (predicción derivada de $\mathrm{H} 02$ ).

\section{Material y Métodos}

Se utilizaron 29 cráneos provenientes de 10 cementerios prehispánicos del valle de Azapa, Arica, Chile (Figura 1), depositados en el Museo Nacional de Historia Natural, Santiago (Colección Blanco Encalada) ${ }^{1}$ (Anexo 1), correspondientes a los períodos Arcaico, Medio, Intermedio Tardío y Tardío $^{2}$ (13 mujeres y 16 hombres, de $24,7 \pm 10,3$ y 33,8 \pm 7,4 años de edad, respectivamente) (Tabla 1), e imágenes radiográficas de 30 pacientes adultos del Servicio Dento Máxilo-Facial del Hospital Clínico de la Universidad de Chile (19 mujeres y 11 hombres, de 30,4 $\pm 15,7$ y $34,7 \pm 11,4$ años de edad, respectivamente), cuyo sexo y edad fueron determinados a partir de las respectivas fichas clínicas. Se incluyó la muestra de cráneos actuales para conocer el grado de dispersión que exhibe la variación general de la forma en ausencia de cualquier tipo de deformación intencional, sirviendo a la vez como control externo de la muestra de cráneos arqueológicos no deformados.

La asociación cultural y cronológica de la muestra de cráneos prehispánicos se determinó en base a las evidencias arqueológicas y dataciones 


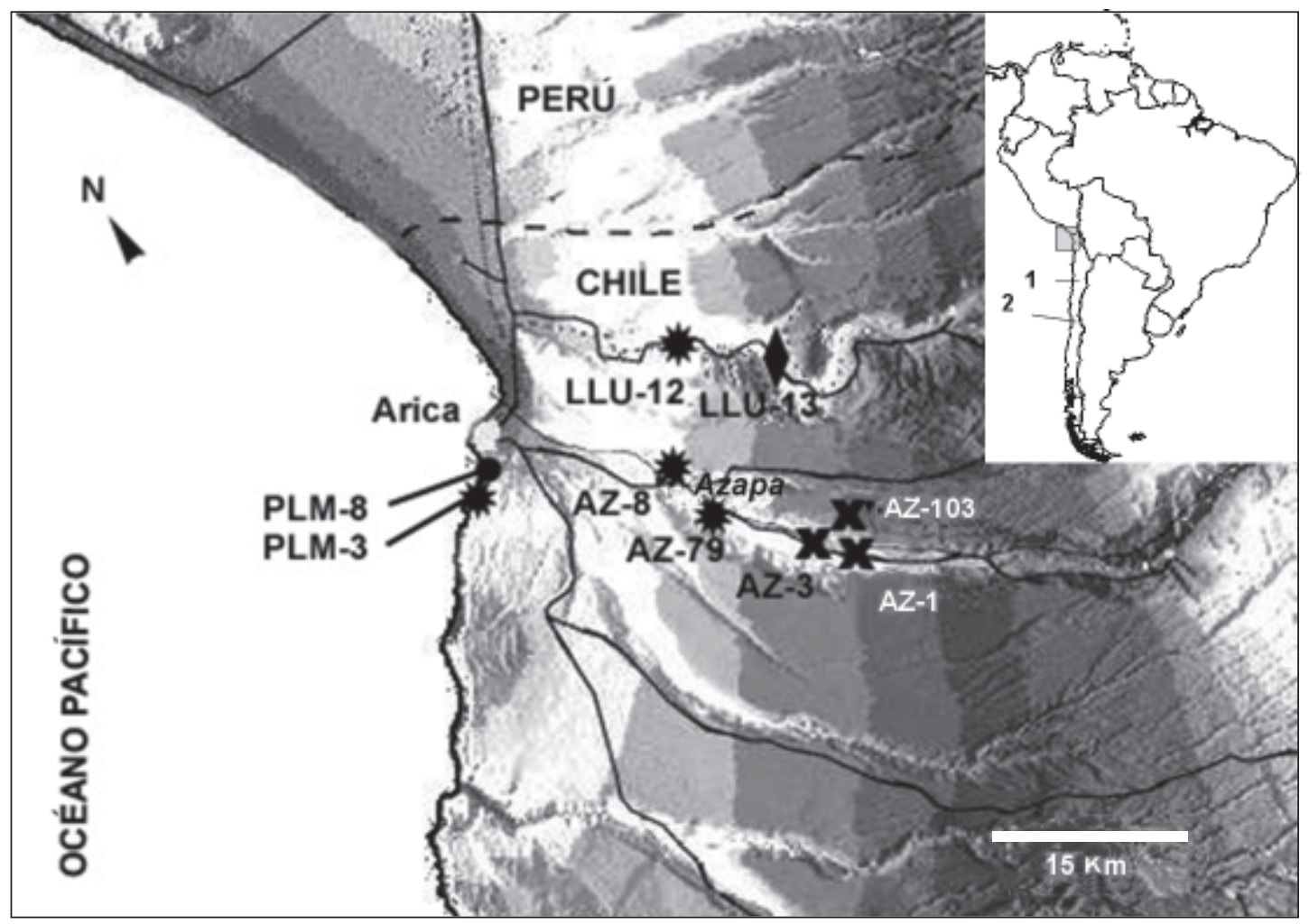

Figura 1. Ubicación de los sitios arqueológicos de los valles de Azapa y Lluta de los períodos Arcaico (•), Medio ( $\mathbf{X})$, Intermedio Tardío ( ) y Tardío ( ) utilizados en este estudio (recuadro superior derecho). La ubicación del sitio de Pica-8 (1) y de Santiago (2) se muestran en el recuadro superior derecho.

Location of the archaeological sites of the Azapa and Lluta, Valleys from the Archaic $(\bullet)$, Middle ( $\mathbf{X})$, Late Intermediate (*) and Late ( ) periods used in this study (upper right inset). The location of the Pica-8 (1) and Santiago (2) sites is shown in the upper right inset.

Tabla 1. Composición de la muestra por período, sexo y tipo de deformación de los cráneos analizados en el presente estudio $(\mathrm{CE}=$ circular erecta, $\mathrm{CO}=$ circular oblicua, $\mathrm{TE}=$ tabular erecta, $\mathrm{TO}=$ tabular oblicua,

$\mathrm{ND}=$ sin deformación intencional, según Dembo e Imbelloni 1938).

Sample of the skulls analysed in the present study by chronological period, sex and type of intentional deformation $(C E=$ Circular erected,$C O=$ Circular oblique, $T E=$ Tabular erected,$T O=$ Tabular oblique, $N D=$ Non deformed, according to Dembo and Imbelloni 1938).

\begin{tabular}{|c|c|c|c|c|c|c|c|c|}
\hline Período & Total & Masculino & Femenino & ND & $\mathrm{CE}$ & $\mathrm{TE}$ & TO & $\mathrm{CO}$ \\
\hline Arcaico & 7 & 3 & 4 & 5 & & 1 & & 1 \\
\hline Medio & 7 & 6 & 1 & 1 & 1 & 2 & 1 & 2 \\
\hline Intermedio Tardío & 13 & 6 & 7 & 1 & 3 & 3 & & 6 \\
\hline Tardío & 2 & 1 & 1 & & 1 & 1 & & \\
\hline Total Arqueológicos & 29 & 16 & 13 & 7 & 5 & 7 & 1 & 9 \\
\hline Total Actuales & 30 & 11 & 19 & 30 & & & & \\
\hline Total & 59 & 27 & 32 & 37 & 5 & 7 & 1 & 9 \\
\hline
\end{tabular}


disponibles para cada sitio en el registro del $\mathrm{Mu}-$ seo Nacional de Historia Natural, Santiago (Colección Blanco Encalada). El sexo se determinó utilizando indicadores morfoscópicos como el desarrollo de la protuberancia iniana, de las líneas nucales, de la glabela, de la arcada cigomática y de las apófisis mastoideas. Para establecer la edad de estos especímenes se analizó el cráneo seco y sus respectivas radiografías en vistas lateral, frontal y mandibular panorámica, y se consideró la erupción del tercer molar, la fusión de la sincondrosis esfeno-occipital, la presencia de facetas de atrición dentaria, el grado de desarrollo radicular, la exostosis del ángulo mandibular y el cierre de la sutura alveolar.

Las radiografías se tomaron con distancia focoplaca de $1,5 \mathrm{~m}$ y empleo de cefalostato en un equipo Siemens (Nanodor-2). Las condiciones de exposición fueron de $75 \mathrm{Kv}, 14 \mathrm{~mA}$ y 0,6 seg en los individuos actuales y de $60 \mathrm{Kv}, 15 \mathrm{~mA}$ y $0,5 \mathrm{seg}$ en los cráneos arqueológicos, debido a la menor can- tidad de tejidos blandos presentes en los últimos. En ambos casos se ocupó un tiempo de revelado de $20 \mathrm{seg}$ a $23^{\circ} \mathrm{C}$. Las fotografías se tomaron en negatoscopio estándar con una cámara digital Fuji FinePix 6900 a distancia y luminosidad fijas.

El registro radiográfico de la muestra arqueológica develó claramente los hitos anatómicos craneanos ocultos por remanentes bioculturales (Figura 2). Para diagnosticar el tipo de deformación e inferir la técnica usada para obtenerla, se analizó individualmente cada ejemplar, y sus radiografías, lateral y frontal. Esta última permitió, además, distinguir el perfil de la norma occipital.

Se ocupó como referencia la clasificación basada en tipos tabulares y circulares, ambos con sus variantes erecta y oblicua, propuesta por Imbelloni (1925) y Dembo e Imbelloni (1938), y su interpretación respecto de los estudios de Soto-Heim (1987). Si bien se han propuesto otras clasificaciones basadas en índices craneométricos (Stewart 1943; Stewart y Newman 1963), en mediciones

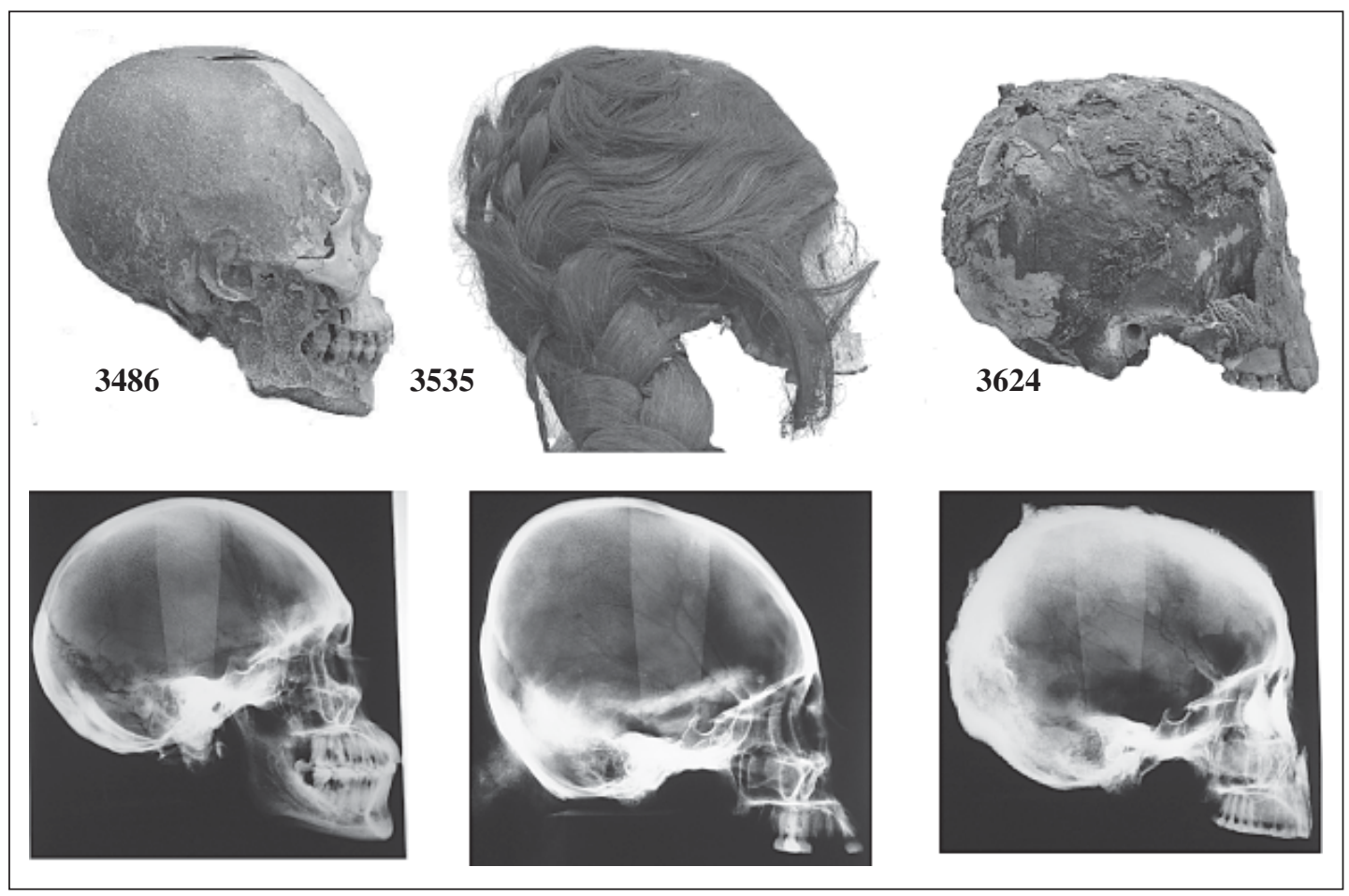

Figura 2. Muestra representativa de los cráneos arqueológicos (fila superior) utilizados en este estudio, depositados en la Colección Manuel Blanco Encalada del Museo Nacional de Historia Natural, Santiago. Las radiografías (fila inferior) revelan las estructuras anatómicas ocultas por restos de piel (MNHN-3486), cabellera (MNHN-3535) y máscara de barro (MNHN-3624).

A representative sample of the archaeological crania (upper row) used in this study, deposited in the Manuel Blanco Encalada Collection of the Museo Nacional de Historia Natural, Santiago. The radiographs (lower row) reveals the anatomical structures hidden by the remains of skin (MNHN-3486), hair (MNHN-3535) and mud mask (MNHN-3624). 
faciales (Soto-Heim 1987) y en criterios de tipología cultural y geográfica (Weiss 1961, 1962), la clasificación de Dembo e Imbelloni (1938) es la de mayor uso en los estudios biológicos sobre deformación intencional en cráneos provenientes del área centro surandina (Cocilovo 1994; Varela y Cocilovo 2002).

En las radiografías se definieron 15 hitos anatómicos homólogos (Tabla 2) que cubren las regiones facial y neural del cráneo en vista lateral derecha (Figura 3). El registro de los hitos se realizó con el programa tpsDig v. 1.31 (Rohlf 2001). Las diferencias debidas a rotación, traslación y escala fueron eliminadas mediante análisis de Procusto según algoritmo del programa tpsRelwarp (v 1.35 Rohlf 2003a) ${ }^{3}$. Las matrices resultantes de este análisis fueron proyectadas como puntos únicos a un bivariado formado por ejes ortogonales, tangentes al espacio morfométrico de Kendall (1984) (análisis de relative warps). Estos ejes (relative warps) son análogos a los componentes principales del análisis multivariado, se obtienen de los vectores propios de los componentes de la forma resultantes del análisis de Procusto (partial warps) y resumen, en orden decreciente, la varianza total acumulada por dichos componentes. En el punto $\mathrm{x}=0$, $\mathrm{y}=0$ de estos ejes se localiza la configuración de consenso que sirve de referencia para visualizar la dirección y magnitud del cambio morfométrico en los especímenes previamente proyectados desde el espacio de Kendall. La visualización del patrón de variación de la forma se obtuvo aplicando la función de placa delgada (thin-plate spline) en los valores extremos positivos y negativos de cada eje, y se representa como una grilla cartesiana que se contrae o expande según la magnitud y dirección que adopta el vector que afecta la forma del cráneo, tanto en sus ejes mayores (cambio uniforme) como en regiones anatómicas altamente localizadas (cambio no uniforme).

Tabla 2. Nombre, definición anatómica y tipo de hitos (sensu Bookstein) utilizados en este estudio (ubicación en Figura 3)

(*Pseudohitos, definidos geométricamente como la proyección sobre la bóveda del punto medio entre dos hitos anatómicos).

Name, anatomical definition and type (sensu Bookstein) of the landmarks used in this study (see Figure 3 for location) (*Pseudolandmarks, defined geometrically as the projection onto the vault of the middle point between two anatomical landmarks).

\begin{tabular}{|c|c|c|c|}
\hline Hito & Nombre & Definición anatómica & Tipo \\
\hline 1 & Nasión & $\begin{array}{l}\text { Intersección de la sutura fronto-nasal con el plano medio-sagital } \\
\text { (Buikstra y Ubelaker 1994) }\end{array}$ & I \\
\hline 2 & Glabela & $\begin{array}{l}\text { Punto más anterior de la línea media del hueso frontal, } \\
\text { generalmente ubicado sobre la sutura fronto-nasal (Buikstra y Ubelaker 1994) }\end{array}$ & III \\
\hline 3 & Bregma & $\begin{array}{l}\text { Punto de la línea media del ectocráneo donde se intersectan las suturas } \\
\text { coronal y sagital (Buikstra y Ubelaker 1994) }\end{array}$ & I \\
\hline 4 & Frontal & $\begin{array}{l}\text { Punto construido geométricamente por la perpendicular, en el punto medio, } \\
\text { de la línea recta que une Glabela y Bregma }\end{array}$ & $*$ \\
\hline 5 & Lambda & $\begin{array}{l}\text { Punto de la línea media del ectocráneo donde se intersectan las suturas } \\
\text { sagital y lambdoidal (Buikstra y Ubelaker 1994) }\end{array}$ & I \\
\hline 6 & Bóveda & $\begin{array}{l}\text { Punto construido geométricamente por la perpendicular, en el punto medio, } \\
\text { de la línea recta que une Bregma y Lambda }\end{array}$ & $*$ \\
\hline 7 & Inión & $\begin{array}{l}\text { Punto ubicado en la base de la protuberancia occipital externa, correspondiente } \\
\text { a la intersección del plano medio sagital con un trazo tangente a la convexidad } \\
\text { más superior de las líneas nucales superiores derecha e izquierda (Bass 1987) }\end{array}$ & II \\
\hline 8 & Ophistion & Punto de la línea media del margen posterior del foramen magno (Bass 1987) & I \\
\hline 9 & Basión & Punto de la línea media del margen anterior del foramen magno (Bass 1987) & I \\
\hline 10 & Apófisis clinoides posterior & Punto más ántero-superior de las apófisis clinoides posteriores & III \\
\hline 11 & Pterigoideo & Punto más póstero-superior de la fosa ptérigo-palato-maxilar & III \\
\hline 12 & Espina nasal posterior & Punto más posterior de la bóveda palatina & I \\
\hline 13 & Punto A & Punto más profundo de la concavidad anterior del maxilar & III \\
\hline 14 & Espina nasal anterior & Punto más anterior del hueso maxilar en la base de la escotadura nasal anterior & I \\
\hline 15 & Suborbitario & Punto más inferior del reborde orbitario & I \\
\hline
\end{tabular}




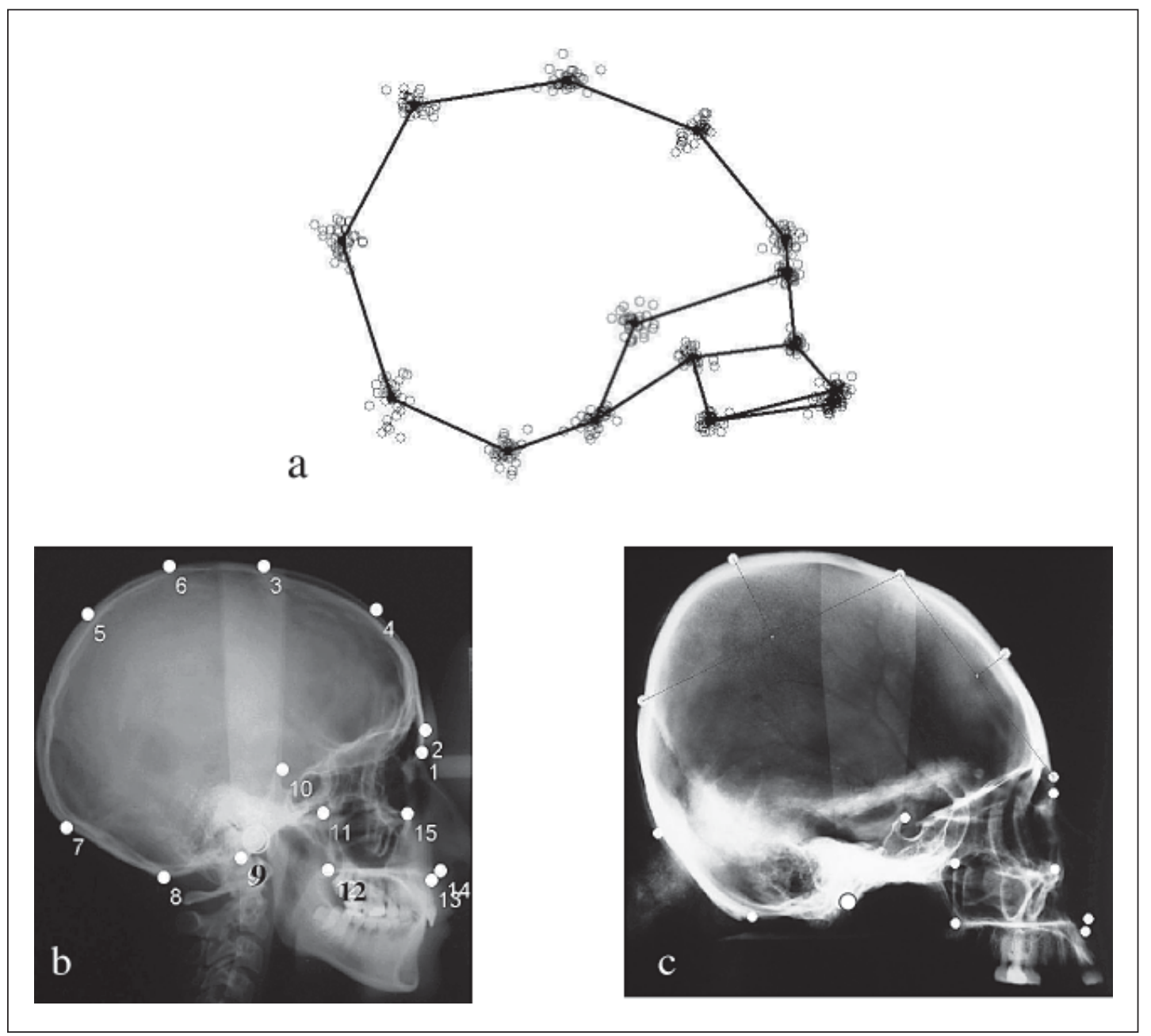

Figura 3. (a) Configuración de consenso con la distribución de los hitos de los especímenes arqueológicos y modernos, (b) hitos de morfocoordenadas en un espécimen moderno, y (c) arqueológico (3535) (definición anatómica en Tabla 2).

(a) Consensus configuration with the distribution of the landmarks for the archaeological and modern specimens, $(b)$ morphocoordinate landmarks in a modern specimen and (c) in an archaeological specimen (3535) (anatomical definition in Table 2).

Las hipótesis estadísticas de ausencia de diferencia entre grupos (cráneos arqueológicos no deformados, arqueológicos deformados y actuales) se pusieron a prueba mediante la regresión de los componentes de la forma contra vectores de variables categóricas $(0,1,2 \ldots)$ para sexo, tipo de deformación y origen cronológico de las muestras (programa tpsReg v. 1.28) (Rohlf 2003b), y fueron evaluadas con la prueba de F según Goodall. Además, se llevó a cabo un análisis de agrupamiento por UPGMA de las distancias de Procusto de las configuraciones de consenso de cada grupo. Con el fin de verificar la robustez de la clasificación a priori de los grupos se realizó un análisis discriminante utilizando los componentes de la forma que explican la mayor varianza acumulada (para $\mathrm{RW}_{1}$ $+\mathrm{RW}_{\mathrm{n}+1}$ 90\%). La contr ibución relativa del componente uniforme $u$ a la variación general de la forma (uniforme + altamente localizada o no uniforme) se calculó según Loy et al. (1998). Como estimador de tamaño geométrico de cada espécimen se utilizó el tamaño del centroide, igual a la raíz cuadrada de la suma de las distancias euclídeas al cuadrado que hay entre cada hito y el centro geométrico (centroide) de las coordenadas de hitos de cada espécimen (Bookstein 1989). 


\section{Resultados}

La inspección visual de las radiografías craneo-faciales reveló el patrón general de variación de la forma del cráneo en las muestras arqueológicas en estudio. La gran mayoría de los ejemplares deformados evidenció la acción de vendajes o amarras flexibles aplicadas entre las regiones frontal y occipital o lambdoidea, con inclinación variable respecto al plano de Frankfurt que, según la clasificación propuesta por Dembo e Imbelloni (1938), correspondería al tipo circular, en sus variantes tanto erecta como oblicua (Figura 4) (Anexo 1) (Espoueys 2003). Además, en los restantes ejemplares deformados se registraron un cráneo tabular oblicuo por tablillas flotantes (3564), dos cráneos tabulares erectos por cuna $(5 \mathrm{~B}, 3535)$ y un cráneo tabular erecto (3634) que presenta colocado en posición un deformador no descrito antes, compuesto por un vendaje en forma de turbante, pero fuertemente inclinado respecto al plano sagital $\left(40^{\circ}\right.$ a $45^{\circ}$ ), sostenido en esa posición por pequeños rellenos textiles asimétricos, y que como consecuencia presenta una marcada plagiocefalia parieto-occipital.

Por otra parte, se observó el progresivo aumento de la proporción de tipos resultantes de la práctica de deformación intencional durante los períodos cronológicos registrados (Figura 4). El análisis exploratorio de los datos mostró que la principal variación de la forma, correspondiente a un $31,1 \%$ de la varianza total (RW1), ocurrió en el orden erectos-no deformados-oblicuos, y se expresó morfológicamente en la contracción del cráneo a nivel de las regiones lambdo-supraoccipital y frontobregmal, y en el aumento del ángulo de la base de cráneo, definido por los hitos nasión, apófisis clinoides posterior, basión (Figura 5, eje x). El segundo componente de la forma (RW2) explicó el $20,7 \%$ de la varianza total y mostró el mayor cambio vectorial en el sentido horizontal-oblicuo-vertical (obélico), manifestado en la elevación del maxilar anterior, la retracción del arco frontal y el aumento del ángulo de la base de cráneo (Figura 5, eje y). La varianza explicada por los 24 restantes componentes de la forma resultó significativamente menor a la de los dos primeros, y no fue graficada $(\mathrm{RW} 3=12,2 \%$, RW4 $=8,3 \%$, RW5 $+\mathrm{RW} 26=27,7 \%$ ).

En los cráneos arqueológicos no deformados el análisis de regresión de los componentes de la variación general de la forma (uniforme y no uniforme) mostró la ausencia de diferencias significativas respecto del sexo y el período (Tabla 3). En consecuencia, en los análisis posteriores estos cráneos se agruparon bajo una misma unidad de comparación. Por su parte, en los cráneos deformados intencionalmente se registraron diferencias altamente significativas en la variación general de la forma debido al efecto del tamaño del centroide y de la deformación ( $\mathrm{p}<0.001)$, tanto cuando se tomaron en cuenta las variantes de cada tipo según Imbelloni (tabular erecta y oblicua, y circular erecta y oblicua) como cuando se consideró sólo el tipo de deformación (circulares y tabulares). Al comparar los cráneos arqueológicos deformados y no deformados el análisis de regresión mostró diferencias significativas entre ambos grupos debidas al sexo, el tamaño del centroide y la deformación intencional (Tabla 3). Sin embargo, cuando se eliminó del análisis el componente uniforme las únicas diferencias significativas se debieron al efecto del tamaño del centroide. Respecto de las clasificaciones entre grupos (sexo, período cronológico y tipo de deformación) el análisis discriminante mostró coincidencias entre el $70 \%$ y $100 \%$ con lo esperado a priori según los antecedentes arqueológicos (Tabla 5).

El análisis de regresión de los cráneos actuales mostró diferencias estadísticamente significativas explicadas por el tamaño del centroide, así como por el dimorfismo sexual de los hitos anatómicos que cubren tanto la región de la cara como el cráneo en su conjunto (Tabla 4). Respecto de la robustez de la discriminación del sexo en estos especímenes, la concordancia de la clasificación a priori con la clasificación obtenida al usar las variables morfométrico-geométricas (relative warps) fue estadísticamente significativa, con valores porcentuales máximos de coincidencia entre lo observado y lo esperado superiores al $80 \%$ (Tabla 5).

La regresión de los componentes de la variación de la forma del cráneo contra el sexo, el tamaño del centroide y el período cronológico mostró diferencias estadísticamente significativas respecto de estas tres variables entre los cráneos arqueológicos y actuales, siendo particularmente alta la contribución del componente uniforme de la variación de la forma debida a las diferencias entre períodos (Tabla 4). En este caso, la concordancia entre los valores observados y esperados fue superior al 90\% (Tabla 5). 


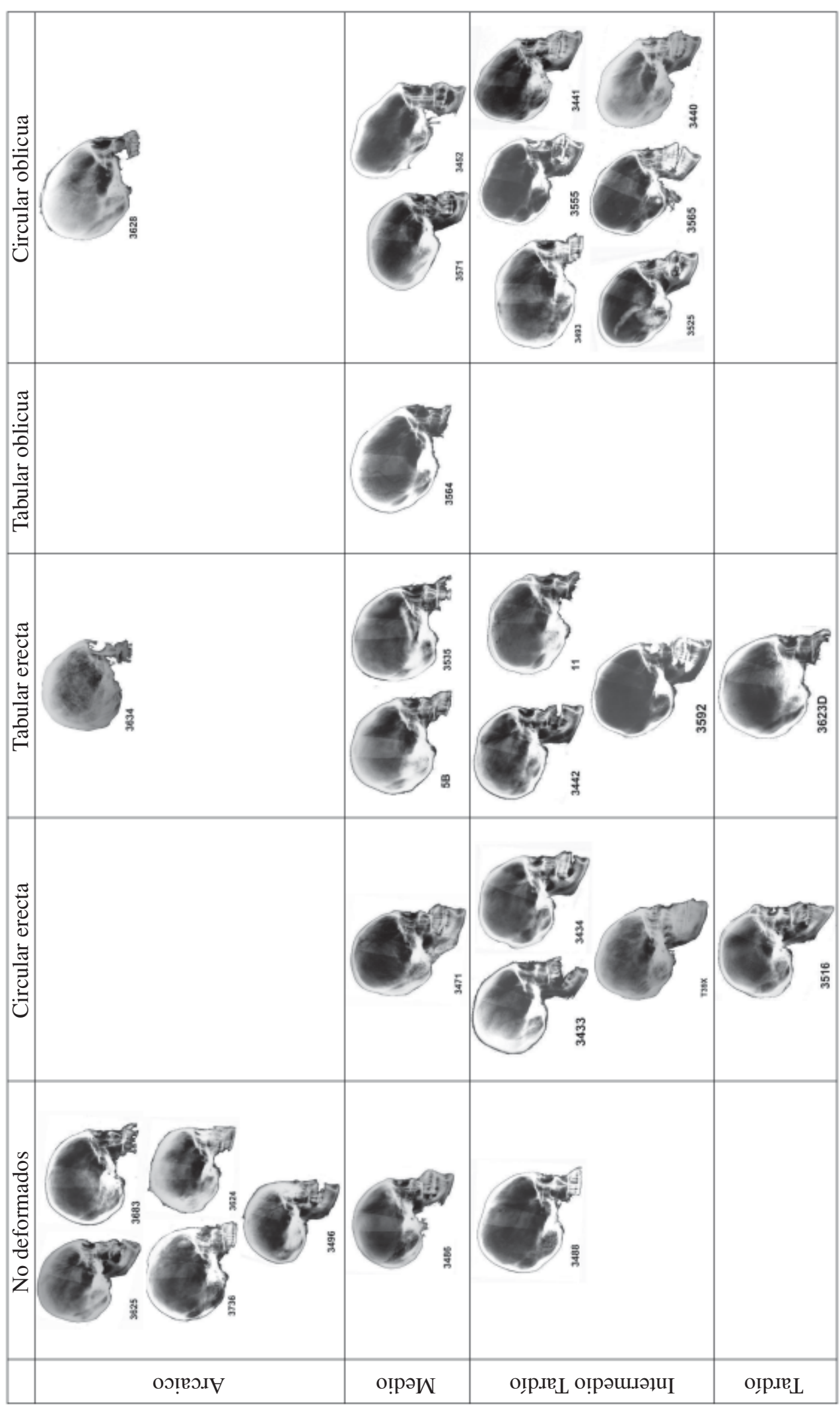

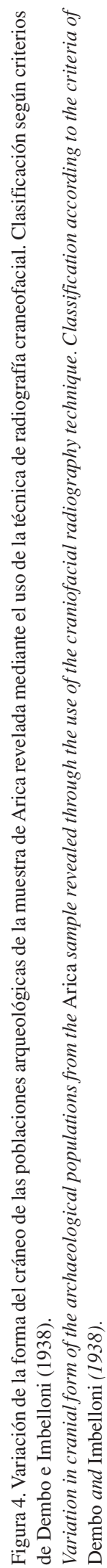




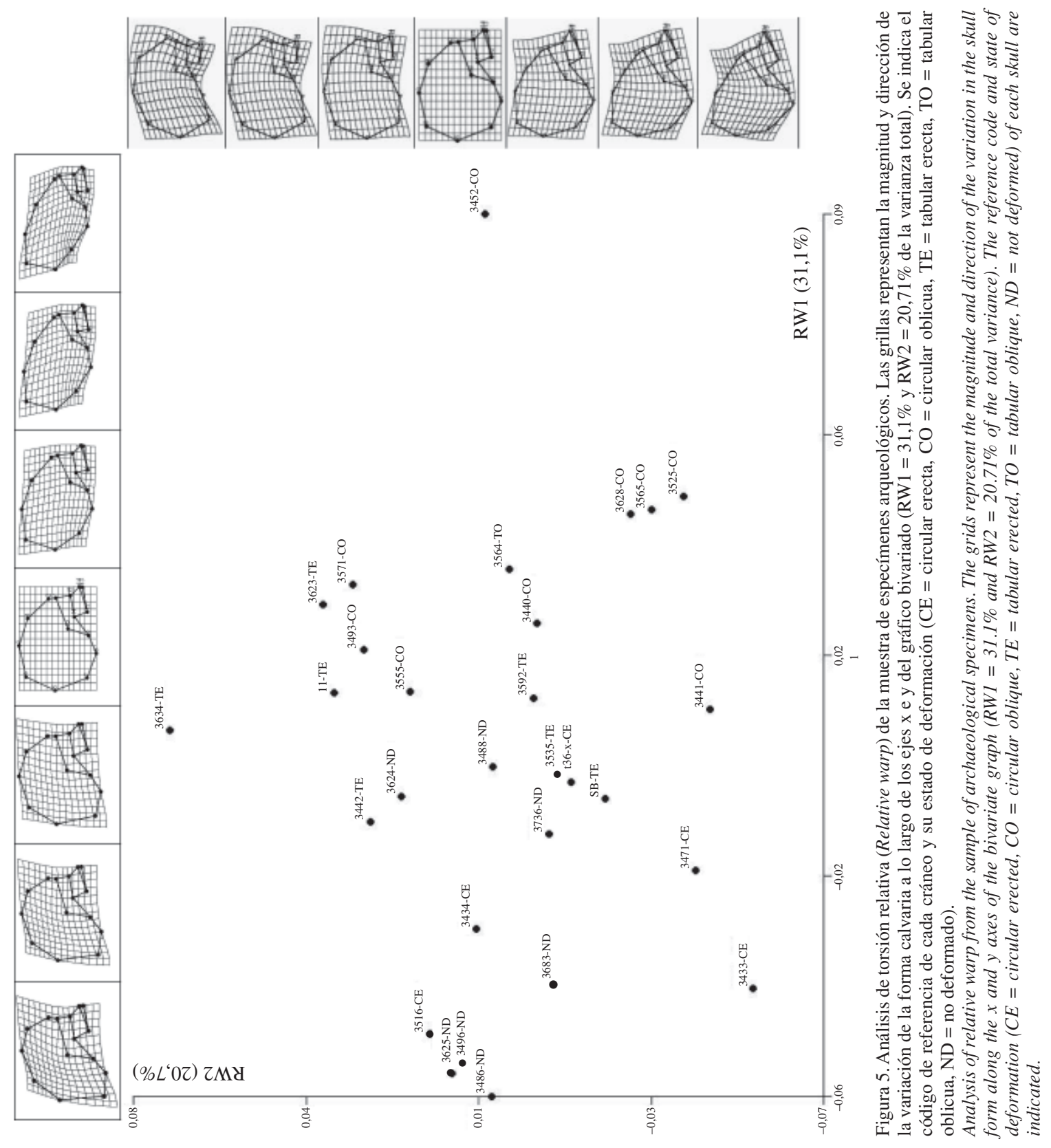


Tabla 3. Efecto de las variables biológicas (sexo, tamaño) y culturales (período, deformación intencional) sobre los componentes de la variación de la forma del cráneo en las muestras arqueológicas de Arica (gl, grados de libertad; p, nivel de significación (en negrilla valores de p estadísticamente significantes); UNI, contribución del componente uniforme a la variación total de la forma; PW, contribución del componente no uniforme, *Tabular erecta, tabular oblicua, circular (variantes erecta y oblicua), **Tabular, circular, según Dembo e Imbelloni 1938).

Effect of biological (sex, size), and cultural (chronological period, type of intentional deformation) variables on the skull shape variation components in the archaeological sample from Arica ( $\mathrm{gl}$, degree of freedom; , level of statistical significance (statistically significant p values in bold); UNI, contribution of the uniform component of overall shape variation; PW, non uniform shape components, *Tabular erected, Tabular oblique, Circular (erected and oblique subtypes), **Tabular and circular, according to Dembo and Imbelloni 1938).

\begin{tabular}{|c|c|c|c|c|c|c|c|c|}
\hline Muestra & $\mathrm{N}$ & Efecto & Goodall & $\mathrm{gl}_{1}$ & $\mathrm{gl}_{2}$ & $\mathrm{p}$ & $\%$ varianza & $\% \mathrm{UNI}$ \\
\hline \multirow[t]{7}{*}{ No deformados } & & $U N I+P W$ & & & & & & \\
\hline & 7 & Sexo & 1,1832 & 26 & 130 & 0,265 & 80,88 & 4,00 \\
\hline & 7 & T. centroide & 1,4219 & 26 & 130 & 0,103 & 77,88 & 1,68 \\
\hline & 7 & $\begin{array}{l}\text { Período } \\
P W\end{array}$ & 0,5576 & 26 & 130 & 0,958 & 89,98 & 0,07 \\
\hline & 7 & Sexo & 0,9453 & 24 & 120 & 0,542 & 84,11 & \\
\hline & 7 & T. centroide & 1,3156 & 24 & 120 & 0,169 & 79,19 & \\
\hline & 7 & Período & 0,5537 & 24 & 120 & 0,953 & 90,05 & \\
\hline \multirow[t]{11}{*}{ Deformados } & & $U N I+P W$ & & & & & & \\
\hline & 22 & Sexo & 0,9213 & 26 & 520 & 0,579 & 95,60 & 0,59 \\
\hline & 22 & T. centroide & 3,5458 & 26 & 520 & $<0,001$ & 84,97 & 8,77 \\
\hline & 22 & Período & 0,4630 & 26 & 520 & 0,990 & 97,74 & 0,13 \\
\hline & 22 & Deformación (variante)* & 2,4666 & 26 & 520 & $<0,001$ & 89,04 & 5,18 \\
\hline & 22 & $\begin{array}{l}\text { Deformación (tipo)** } \\
P W\end{array}$ & 1,9054 & 26 & 520 & 0,005 & 91,32 & 1,51 \\
\hline & 22 & Sexo & 0,7986 & 24 & 480 & 0,740 & 96,16 & \\
\hline & 22 & T. centroide & 1,6437 & 24 & 480 & 0,029 & 92,42 & \\
\hline & 22 & Período & 0,4361 & 24 & 480 & 0,992 & 97,87 & \\
\hline & 22 & Deformación (variante) & 1,3576 & 24 & 480 & 0,121 & 93,66 & \\
\hline & 22 & Deformación (tipo) & 1,5786 & 24 & 480 & 0,041 & 92,70 & \\
\hline \multirow[t]{13}{*}{ No def. y Def. } & & $U N I+P W$ & & & & & & \\
\hline & 29 & Sexo & 1,7952 & 26 & 702 & 0,009 & 93,77 & 2,01 \\
\hline & 29 & T. centroide & 4,8594 & 26 & 702 & $<0,001$ & 84,78 & 10,22 \\
\hline & 29 & Período & 0,7805 & 26 & 702 & 0,775 & 97,20 & 1,19 \\
\hline & 29 & Deformación (variante) & 4,3864 & 26 & 702 & $<0,001$ & 86,06 & 11,44 \\
\hline & 29 & Deformación (tipo) & 1,9934 & 26 & 702 & 0,003 & 93,14 & 1,93 \\
\hline & 29 & $\begin{array}{l}\text { Deformados vs No deformados } \\
P W\end{array}$ & 3,0797 & 26 & 702 & $<0,001$ & 89,78 & 7,46 \\
\hline & 29 & Sexo & 1,2269 & 24 & 648 & 0,210 & 95,66 & \\
\hline & 29 & T. centroide & 1,8966 & 24 & 648 & 0,006 & 93,45 & \\
\hline & 29 & Período & 0,4518 & 24 & 648 & 0,990 & 98,36 & \\
\hline & 29 & Deformación (variante) & 1,1557 & 24 & 648 & 0,277 & 95,91 & \\
\hline & 29 & Deformación (tipo) & 1,4417 & 24 & 648 & 0,080 & 94,94 & \\
\hline & 29 & Deformados vs No deformados & 0,9857 & 24 & 648 & 0,483 & 96,49 & \\
\hline
\end{tabular}

El examen del patrón de variación de las grillas de deformación relativa a lo largo del primer eje de los componentes de la forma (relative warp 1) reveló (a) una clara separación entre los cráneos actuales y arqueológicos, y (b) la región más afectada en la vista lateral por la eventual aplicación de los aparatos deformadores reside en la región occipital de los cráneos arqueológicos y está ubicada entre lambda e inión (Figura 6). En concordancia con lo anterior, los hitos 5 (lambda) y 7 (inión) son los que muestran la mayor dispersión en la configuración de consenso de los cráneos actuales y arqueológicos (Figura 3a). La inspección de las grillas reveló, además, que en la muestra arqueológica las modificaciones del ectocráneo covarían en dirección y magnitud con cambios que ocurren en la base de cráneo (Figura 5). Expresión de lo anterior es el elevamiento de la apófisis clinoides posterior, generando una disminución del ángulo base de cráneo, conformado por los hitos 
Tabla 4. Análisis del efecto de las variables biológicas (sexo, tamaño) y culturales (deformación, período) sobre los componentes de la variación de la forma del cráneo y de la cara en las poblaciones arqueológicas y actuales (gl, grados de libertad; p, nivel de significación) (en negrilla valores de p estadísticamente significantes).

Analysis of the effect of the biological and cultural variables on the cranial and facial shape variation components in the archaeological and modern samples ( $\mathrm{gl}$, degree of freedom; $p$, level of statistical significance) (statistically significant $p$ values in bold).

\begin{tabular}{|c|c|c|c|c|c|c|c|c|}
\hline Muestra & $\mathrm{N}$ & Efecto & F de Goodall & $\mathrm{gl}_{1}$ & $\mathrm{gl}_{2}$ & $\mathrm{p}$ & $\%$ varianza & $\% \mathrm{UNI}$ \\
\hline \multirow[t]{10}{*}{ Chilenos actuales } & \multirow[t]{10}{*}{30} & $U N I+P W$ & & & & & & \\
\hline & & Sexo (Cráneo) & 1,92 & 26 & 728 & 0,0040 & 93,60 & 0,83 \\
\hline & & Sexo (Cara) & 2,67 & 12 & 336 & 0,0020 & 91,33 & 2,65 \\
\hline & & T. centroide (Cráneo) & 17,67 & 26 & 728 & $<0,001$ & 61,33 & 10,53 \\
\hline & & T. centroide (Cara) & 0,97 & 12 & 336 & 0,478 & 96,66 & 1,41 \\
\hline & & $P W$ & & & & & & \\
\hline & & Sexo (Cráneo) & 1,67 & 24 & 672 & 0,0235 & 94,38 & \\
\hline & & Sexo (Cara) & 1,85 & 10 & 280 & 0,0518 & 93,81 & \\
\hline & & T. centroide (Cráneo) & 12,86 & 24 & 672 & $<0,001$ & 68,55 & \\
\hline & & T. centroide (Cara) & 0,56 & 10 & 280 & 0,846 & 98,04 & \\
\hline \multirow[t]{14}{*}{ Actuales + Arqueológicos } & \multirow[t]{14}{*}{59} & $U N I+P W$ & & & & & & \\
\hline & & Sexo (Cráneo) & 1,74 & 26 & 1482 & 0,0123 & 97,05 & 0,22 \\
\hline & & Sexo (Cara) & 1,64 & 12 & 684 & 0,0770 & 97,22 & 1,24 \\
\hline & & T. centroide (Cráneo) & 23,97 & 26 & 1482 & $<0,001$ & 70,40 & 5,39 \\
\hline & & T. centroide (Cara) & 2,45 & 12 & 684 & 0,0040 & 95,89 & 0,49 \\
\hline & & Período (Cráneo) & 25,80 & 26 & 1482 & $<0,001$ & 68,91 & 12,57 \\
\hline & & Período (Cara) & 10,22 & 12 & 684 & $<0,001$ & 84,84 & 3,28 \\
\hline & & $P W$ & & & & & & \\
\hline & & Sexo (Cráneo) & 1,61 & 24 & 1368 & 0,0317 & 97,27 & \\
\hline & & Sexo (Cara) & 0,90 & 10 & 570 & 0,5290 & 98,44 & \\
\hline & & T. centroide (Cráneo) & 19,61 & 24 & 1368 & $<0,001$ & 74,41 & \\
\hline & & T. centroide (Cara) & 2,16 & 10 & 570 & 0,0191 & 96,36 & \\
\hline & & Período (Cráneo) & 15,38 & 24 & 1368 & $<0,001$ & 78,82 & \\
\hline & & Período (Cara) & 8,01 & 10 & 570 & $<0,001$ & 87,7 & \\
\hline
\end{tabular}

Tabla 5. Análisis discriminante de los componentes de la forma craneana (RW1 $+\mathrm{RW}_{\mathrm{n}+1}>90 \%$ de la varianza acumulada) de las muestras utilizadas en este estudio (gl, grados de libertad; $\mathrm{p}$, nivel de significación) (en negrilla valores de p estadísticamente significantes).

Discriminant analysis of skull shape components $\left(R W 1+R W_{n+l}>90 \%\right.$ of overall variance) of the samples used in this study ( $\mathrm{gl}$, degree of freedom; $p$, level of statistical significance) (statistically significant $p$ values in bold).

\begin{tabular}{|c|c|c|c|c|c|c|c|c|c|c|}
\hline Muestra & $\mathrm{N}$ & $\begin{array}{l}\text { Variable } \\
\text { (Grupo 1 vs } \\
\text { Grupo 2) }\end{array}$ & $\begin{array}{c}\% \\
\text { Grupo } 1\end{array}$ & $\begin{array}{c}\% \\
\text { Grupo } 2\end{array}$ & $\begin{array}{l}\text { Tolerancia } \\
\text { promedio }\end{array}$ & $\begin{array}{c}\text { Lambda } \\
\text { de } \\
\text { Wilks }\end{array}$ & $\mathrm{F}$ & $\mathrm{gl}_{1}$ & $\mathrm{gl}_{2}$ & $\mathrm{p}$ \\
\hline \multirow[t]{2}{*}{ Arqueológicos } & 29 & $\begin{array}{l}\text { Sexo } \\
\text { (Femenino vs } \\
\text { Masculino) }\end{array}$ & 76,92 & 81,25 & 0,973 & 0,564 & 1,63 & 9 & 19 & 0,176 \\
\hline & 29 & $\begin{array}{l}\text { Deformación } \\
\text { (No Deformados vs } \\
\text { Deformados) }\end{array}$ & 100 & 71,43 & 0,978 & 0,549 & 1,73 & 9 & 19 & 0,150 \\
\hline Actuales & 30 & $\begin{array}{l}\text { Sexo } \\
\text { (Femenino vs } \\
\text { Masculino) }\end{array}$ & 94,74 & 81,82 & 0,943 & 0,458 & 3,10 & 8 & 21 & $<0,05$ \\
\hline $\begin{array}{l}\text { Arqueológicos/ } \\
\text { Actuales }\end{array}$ & 59 & $\begin{array}{l}\text { Período } \\
\text { (Arqueológico vs } \\
\quad \text { Actual) }\end{array}$ & 93,10 & 96,67 & 0,923 & 0,233 & 20,54 & 8 & 50 & $<0,01$ \\
\hline
\end{tabular}



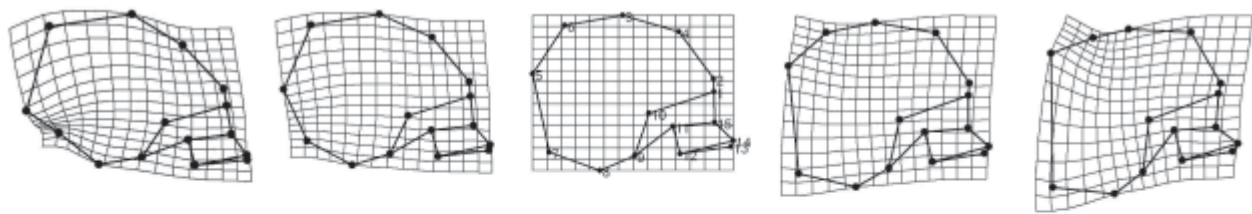

ARQUEOLÓGICOS

ACTUALES

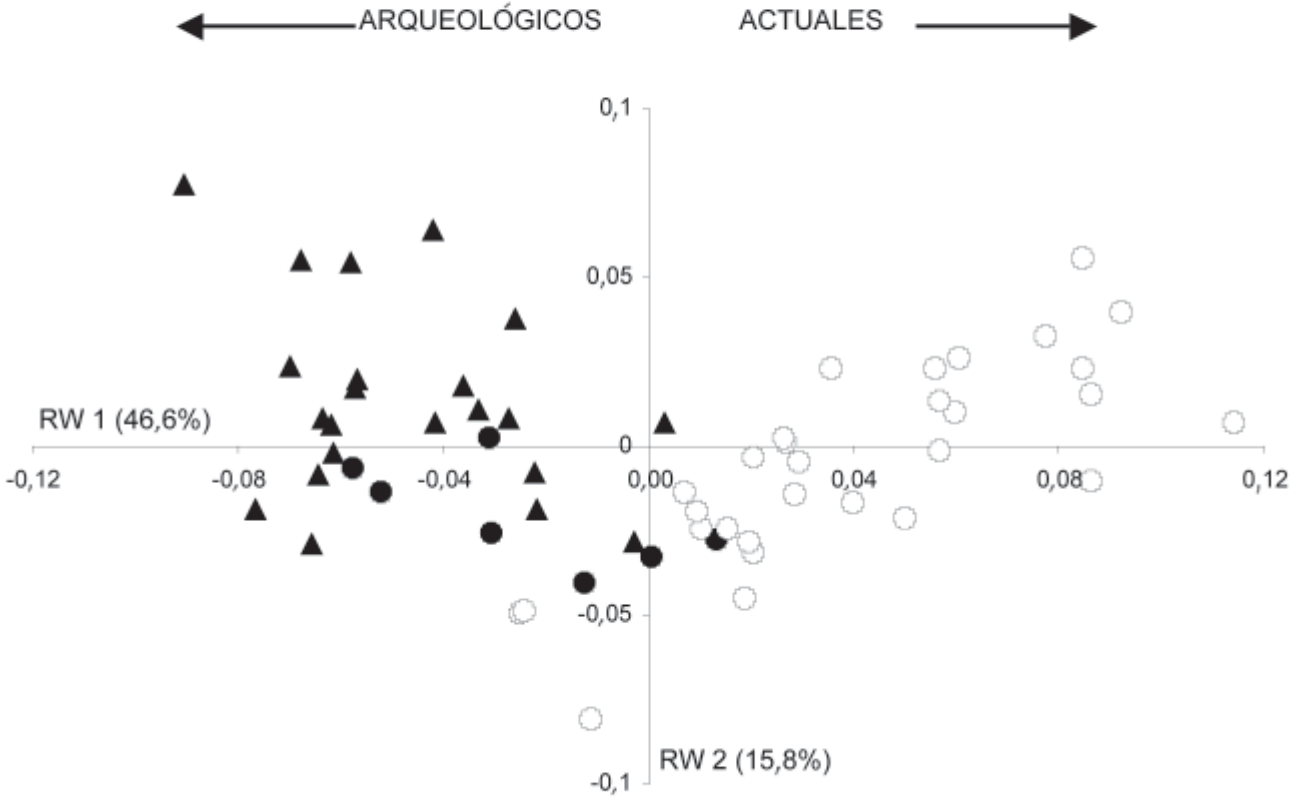

$\Delta$ Arqueológicos deformados • Arqueológicos no deformados Actuales

Figura 6. Análisis de torsión relativa de la muestra de especímenes arqueológicos y contemporáneos. Las grillas representan la magnitud y dirección de la variación de la forma a lo largo del eje que acumula la mayor varianza (RW1 = 46,6\%).

Analysis of relative warp from sample of archaeological and contemporary specimens. The grids represent the magnitude and direction of the variation of the form along the axis that accumula.

nasión, apófisis clinoides posterior y basión (hitos 1, 10 y 9 en Figura 3), y del punto más pósterosuperior de la fosa ptérigo-palato-maxilar (hito 11 en Figura 3), asociada con el descenso de la región bregmiana. De los tres hitos que definen el ángulo base de cráneo, la mayor contribución relativa a esta variación la aportó nasión, seguido de apófisis clinoides posterior y basión (suma de cuadrados $=$ $0,04866,0,00447$ y 0,00357 , respectivamente).

\section{Discusión}

\section{Variación general de la forma del cráneo}

Los resultados sugieren que la variación de los componentes de la forma craneana, en particular en los ejemplares con deformación circular oblicua y tabular oblicua, depende de la magnitud y dirección con que se aplicaron y orientaron originalmente los aparatos deformadores sobre los ejes anatómicos mayores del cráneo. El primero de dichos componentes se manifiesta en una contracción en dirección ántero-posterior, de mayor intensidad que la contracción explicada por el segundo componente, reflejado en la expansión de la región maxilar anterior (eje $\mathrm{x}=\mathrm{RW} 1$ y eje $\mathrm{y}=\mathrm{RW} 2$ en Figura 5). Este último cambio sería expresión vectorial de la presión ejercida por el aparato deformador y concuerda con el cambio que según Dembo e Imbelloni (1938) e Imbelloni (1932) experimenta el eje del sólido deformado en su inclinación respecto del plano de Frankfurt. 
Llama la atención que este aspecto no sea considerado en la mayor parte de los trabajos sobre deformación artificial que utilizan la clasificación de Dembo e Imbelloni (1938) (Munizaga 1964, 1973; Soto-Heim 1987), ya que agrupan bajo un mismo criterio clasificatorio a cráneos que según su grado de inclinación deberían estar separados en tres subgrupos, que corresponden a las distintas inclinaciones del eje del sólido neurocraneal: circular horizontal (3493 y 3571), circular diagonal (3440 y 3452) y circular obélico $(3441,3552,3565$ y 3628$)^{4}$.

Por otro lado, los cráneos arqueológicos no deformados no ocupan un lugar equidistante respecto de los que han sufrido el proceso de deformación, sino que tienden a agruparse con cráneos circulares y tabulares erectos. En buena medida, esto se explicaría porque, siendo el material radiográfico bidimensional por naturaleza, el análisis morfométrico no es capaz de registrar al mismo tiempo los componentes sagital y coronario de la variación de la forma del cráneo.

\section{Distribución de las deformaciones}

La casi nula presencia de deformaciones tabulares oblicuas realizadas con uso de tablillas, revelada por la inspección visual del material radiografiado, sugiere el carácter culturamente ajeno de estos aparatos a la tradición deformadora local representada en la muestra (Figura 4). Se observa, además, que la mayor frecuencia de cráneos con deformaciones extremas o de mayor intensidad deformadora registradas ocurre en los períodos Medio e Intermedio Tardío, y está representada por el tipo circular oblicuo y tabular oblicuo (Figura 4). Sugerentemente, de los siete ejemplares clasificados como tabulares erectos a partir de Dembo e Imbelloni (1938), cuatro de ellos (3442, 11, 3592 y 3623D) corresponden a los tipos definidos como pseudocirculares por Soto-Heim $(1987)^{1}$, debido a que su deformación se obtuvo con el uso de vendajes nucales en lugar de superficies rígidas.

Es importante destacar el progresivo aumento de la diversidad de tipos resultantes de la práctica de deformación intencional durante los períodos cronológicos registrados (Figura 4). Los cráneos pseudocirculares se ubican en los períodos Intermedio Tardío y Tardío y los cráneos tabulares erec- tos aparecen en los períodos Arcaico y Medio, resultantes de la fijación del cuerpo y/o el cráneo del infante a una cuna mediante amarras $(5 \mathrm{~B}, 3535$, 3634). Estos cambios estarían asociados con la disminución en el tiempo de la frecuencia de cráneos no deformados, pues mientras en el período Arcaico éstos representan un 71,4\% del total, en los períodos posteriores su frecuencia disminuye progresivamente $(14,3 \%$ en el período Medio y $7,1 \%$ en el período Intermedio Tardío) (Figura 4). De este modo, los resultados de la inspección visual del material radiográfico sugieren que al aumentar la diversificación étnica en la costa y los valles de la Arica precolombina, también se masifica la deformación intencional y aumenta el grado de intensidad de esta práctica.

\section{Efecto de la deformación intencional sobre los hitos de la cara}

Según los trabajos que ocupan el enfoque antropométrico lineal para conocer la variación craneométrica de las poblaciones prehispánicas de Sudamérica, el efecto de la deformación artificial del cráneo sobre mediciones esplacnocraneanas (por ejemplo ancho frontal mínimo, ancho bicigomático, altura nasal, altura orbital, ancho orbital, ancho palatino) es menos intenso que en el neurocráneo y, en términos prácticos, dichas mediciones podrían considerarse no afectadas por la deformación (Cocilovo 1975, 1978; Rothhammer et al. 1983, 2002). Sin embargo, al analizar una muestra de 350 cráneos colectados de sitios arqueológicos del valle de Azapa que incluía, en igual proporción, tipos tabulares, circulares y no deformados, Rhode (2002) encontró que las mediciones antes mencionadas variaban significativamente en los cráneos deformados intencionalmente.

Aunque se requiere aumentar el número de cráneos telerradiografiados sometidos al análisis de morfometría geométrica, las evidencias obtenidas en el presente trabajo sugieren que el uso de los aparatos deformadores afecta de manera similar tanto a los hitos que cubren la bóveda como a los que están inscritos en la región de la cara, en particular a nivel del frontal y maxilar anterior (hitos 2 y 14 en Figura 4 y serie de grillas en Figura 5). En concordancia con lo anterior, análisis de 
morfometría lineal realizados en muestras de cráneos con deformación intencional provenientes de poblaciones prehispánicas de Norteamérica y del Perú revelan que una de las consecuencias anatómicas de la deformación intencional es el efecto constante que tiene la modificación de la bóveda craneana sobre la cara, consistente, con excepción de la región nasal (Anton 1989), en una expansión en el eje medio-lateral (Cheverud et al. 1992), que involucra el ensanchamiento posterior de la mandíbula (Cheverud y Midkiff 1992) y la platibasis de la base de cráneo (Anton 1989; McNeill y Newton 1965). Estos resultados han sido corroborados recientemente al aplicar técnicas de morfometría geométrica para el análisis de contornos (Friess y Baylac 2003), siendo la magnitud de los cambios que afectan la porción inferior de la cara mayor que la observada en la base de cráneo.

\section{Eventual continuidad morfológica}

Aunque nuestros datos no permiten hacer inferencias fundamentadas sobre el grado de geneticidad que explica el patrón de variación craneana, la continuidad temporal de la morfología de las poblaciones arcaicas y agroalfareras, como también la ausencia de diferencias significativas entre estas últimas, sugieren la eventual ocurrencia de flujo genético entre los enclaves arcaicos de la costa y los agroalfareros posteriores de los valles bajos durante un largo período de tiempo. A similar conclusión llegan los autores que han utilizado caracteres no métricos (Blom et al. 1998; Rothhammer et al. 1984; Sutter y Mertz 2004).

En lo que se refiere a la comparación de los cráneos arqueológicos con los cráneos actuales, el análisis de agrupamiento de las configuraciones de consenso de las muestras separadas según el período cronológico (distancias de Procusto por UPGMA) (Figura 7) mostró continuidad temporal entre períodos (Arcaico $\rightarrow$ Medio $\rightarrow$ Intermedio Tardío $\rightarrow$ Actual). Al respecto, es relevante recordar que las únicas diferencias de la variación de la forma craneana estadísticamente significativas ocurrieron entre las muestras arqueológica y contemporánea, y que las diferencias significativas observadas en la muestra de cráneos arqueológicos se debieron al tipo de deformación y al tamaño del

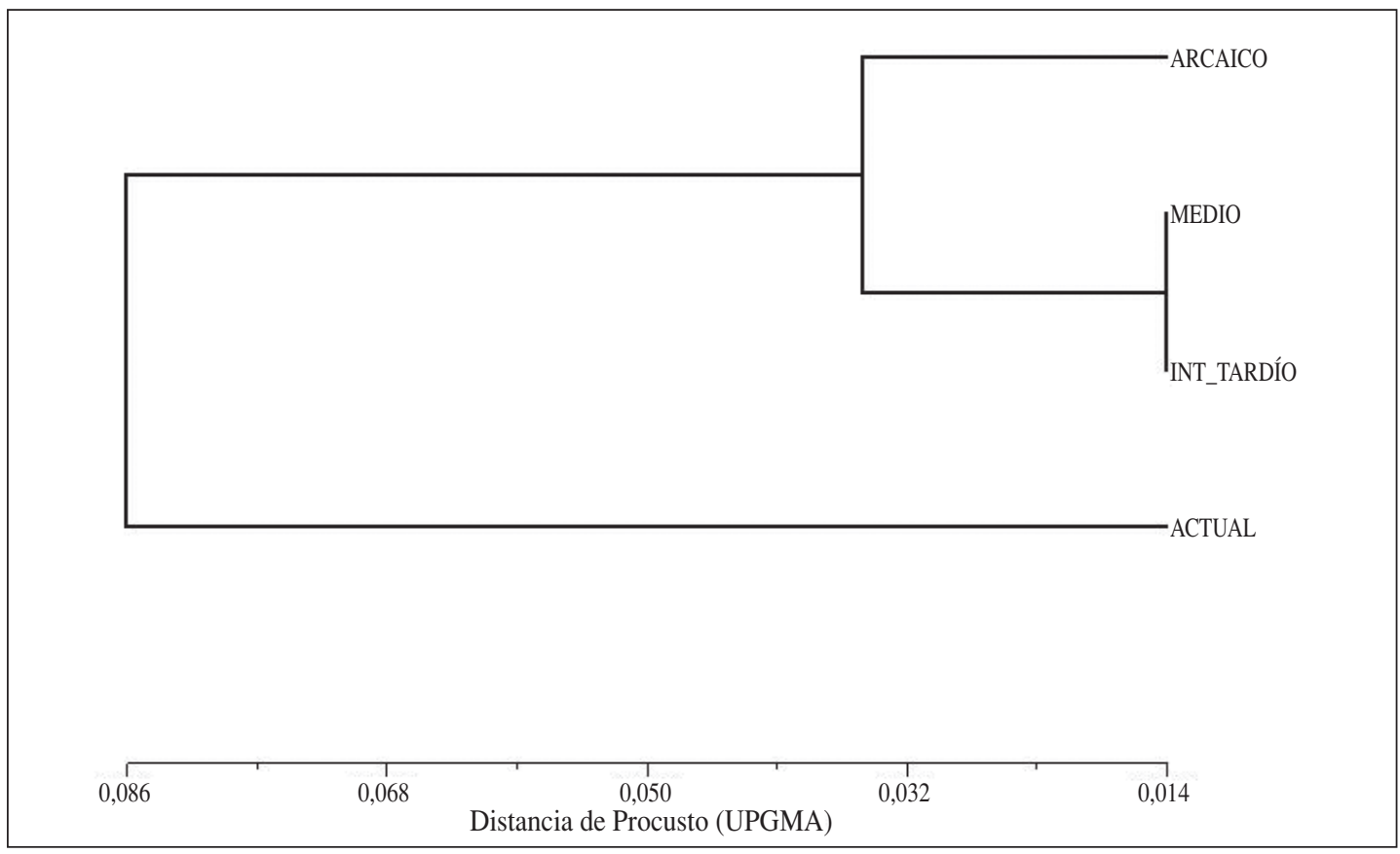

Figura 7. Análisis de agrupamiento de las distancias morfométrico-geométricas (de Procusto) entre las configuraciones de consenso de las muestras utilizadas en este estudio.

Analysis of the grouping of the geometric morphometric distance (from Procusto) between the consensus configurations of the sample used in this study. 
centroide, pero no a la pertenencia a uno u otro período cronológico. Respecto de los cráneos actuales, el hecho de que algunas de sus configuraciones compartan un mismo espacio morfométrico con cráneos arqueológicos no deformados (Figura 6), evidencia que la forma del cráneo en poblaciones humanas actuales no sometidas a deformación intencional corresponde a un fenotipo altamente variable, cuya norma se define desde un punto de vista poblacional antes que tipológico.

Por último, la alta significación estadística de las diferencias debidas al tamaño del centroide en los cráneos actuales sugiere que gran parte de la variación de la forma entre sexos está asociada con la variación escalar de los hitos anatómicos de la cara, y corrobora los resultados obtenidos al aplicar las técnicas de la morfometría "clásica" al estudio del dimorfismo sexual en cráneos humanos (Bass 1987).

Consideramos que la discrepancia de nuestros resultados sobre la afinidad morfológica de estas poblaciones con los obtenidos en los análisis métricos tradicionales (Cocilovo 1994) se explica porque estos últimos emplean estimadores lineales del tamaño. Debido a su naturaleza unidimensional (valores únicos de distancia entre hitos anatómicos), dichos estimadores no permiten la partición ortogonal de los componentes de la forma y el tamaño. El enfoque de la morfometría geométrica utilizado en el presente trabajo (análisis de Procusto de los espacios de Kendall) otorga la posibilidad de realizar tal partición debido a la naturaleza multidimensional de los datos primarios (2 ó 3 dimensiones). De acuerdo con nuestros resultados, las fuentes de variación estadísticamente significativa entre los cráneos de las poblaciones arcaicas y agroalfarera son el tamaño del centroide y la deformación artificial (Tabla 3). Por el contrario, cuando los intentos de partición de la forma respecto del tamaño se han llevado a cabo bajo los supuestos de la morfometría lineal (Spielman 1972), el aporte del tamaño a la variación craneana de estas poblaciones resulta insignificante (Rothhammer et al. 1982). Las diferencias entre los resultados obtenidos por los enfoques lineal y geométrico se explican porque en el primer caso el procedimiento consiste en la transformación geométrica de vectores lineales, mientras que en el segundo se realiza una transformación lineal de datos de naturaleza geométrica.

\section{Aplicación a casos clínicos}

La covariación de los hitos que conforman el ángulo de la base de cráneo con los hitos del ectocráneo, descrito en el presente trabajo como un cambio en dicha angulación, es anatómicamente equivalente a lo observado en poblaciones modernas que sufren malformaciones esqueletales (relación máxilo-mandibular). La experiencia clínica enseña que la disminución del ángulo base de cráneo característico de un biotipo braquicefálico genera una tendencia a la rotación anterior mandibular resultando en un pronunciado prognatismo, mientras que el aumento de este ángulo, propio del biotipo dolicocefálico, está asociado a retrognacia con tendencia a la rotación posterior mandibular (Enlow y Hans 1998). Estos antecedentes sugieren la eventual aplicación de las técnicas de la morfometría geométrica en el área clínica, donde los cráneos arqueológicos afectados por la deformación intencional representarían un "modelo extremo" de los casos de disfunción máxilo-mandibular observados en la población actual. Aunque se ha comenzado a trabajar en estos problemas (González et al. 2004), su estudio es aún incipiente en nuestro país.

El enfoque morfométrico utilizado en este trabajo permitiría, además, conocer las eventuales consecuencias mórbido-traumáticas del proceso de deformación en las poblaciones arqueológicas. Según Allison et al. (1981), la práctica de la deformación artificial no tiene efectos patológicos detectables. No obstante haber observado en muchos casos el cierre prematuro de algunas suturas, estos autores afirman no haber encontrado evidencias que les permitan suponer la ocurrencia de microcefalias u otras alteraciones en el desarrollo craneano de los individuos sometidos a la práctica de deformación intencional. Sin embargo, en poblaciones agroalfareras del valle de Azapa, Gerszten (1993) halló un aumento significativo de la incidencia de huesos intercalares en la región de la sutura lambdoidea de los cráneos deformados artificialmente en comparación con los no deformados, relacionándolo con la necrosis del hueso occipital. En estos casos, la presión del aparato deformador sería responsable del colapso parcial del flujo sanguíneo en la zona donde éste se aplica, lo que alteraría el normal desarrollo encefálico (Gerszten et al. 1998). Estos antecedentes coinciden con lo observado por otros autores en poblaciones prehis- 
pánicas de Norteamérica y Sudamérica respecto del efecto de la deformación intencional en la frecuencia de huesos intercalares, el grado de interdigitación, la presencia de sinostosis y el incremento de la vascularización endocraneal (Anton et al. 1992; O’Loughlin 1996; Ossenberg 1970; White 1966).

En resumen, el uso combinado de la morfometría geométrica y las técnicas radiográficas permite enfrentar problemas clásicos de la antropología física, pero plantea nuevas interrogantes, de interés no sólo para antropólogos y arqueólogos sino que, también, para los especialistas del área clínica y ortodóncica. ¿Tuvo la deformación artificial del cráneo consecuencias traumáticas en la función masticatoria de los individuos afectados por esta práctica? ¿En qué medida los efectos de la deformación artificial sobre el cráneo covarían con la morbilidad ósea y con el polimorfismo craneano preexistente? ¿Hay regresión al estado predeformado cuando el aparato deformador es retirado? son algunas de estas interrogantes.

\section{Conclusiones}

Los resultados obtenidos al aplicar las técnicas de morfometría geométrica nos llevan a concluir que en las poblaciones prehispánicas estudiadas: (1) la variación de los componentes de la forma craneana depende de la magnitud y dirección con que se aplicaron y orientaron originalmente los aparatos deformadores sobre los ejes anatómicos mayores del cráneo, (2) la deformación intencional involucra a los hitos anatómicos del neurocráneo y de la cara, (3) el tipo y variedad de deformación intencional sería independiente del período cronológico durante el cual se llevó a cabo esta práctica, sugiriendo la ocurrencia de una continuidad morfológica entre las poblaciones prehispánicas de la costa y valles de Arica.

Nuevos análisis que incluyan un mayor número de cráneos permitirán contrastar estas conclusiones preliminares, pero es claro que la aplicación de las herramientas de la morfometría geométrica y de la telerradiografía craneofacial facilitan el estudio del patrón de variación de la forma del cráneo en muestras que habitualmente no son utilizadas en los estudios antropométricos de las poblaciones prehispánicas del norte árido de Chile, debido a la presencia de restos de origen biológico y cultural. El presente estudio muestra que el uso de estas técnicas representa un complemento necesario para aumentar el conocimiento sobre las consecuencias bioantropológicas de la práctica de deformación intencional del cráneo en las poblaciones prehispánicas de Arica.

Agradecimientos: Este trabajo fue financiado parcialmente por Proyectos Fondecyt 1020375 (GM) y 1050279 (GM). Agradecemos especialmente la participación de Silvia Quevedo Kawasaki como coinvestigadora en la etapa inicial del Proyecto 1020375, entonces curadora de la Sección de Antropología Física del Museo Nacional de Historia Natural de Santiago, de Julia Flores, Juana Valenzuela y Fernando Martínez por el apoyo técnico para obtener las radiografías del material arqueológico en el Servicio Dento-Máxilo Facial del Hospital Clínico de la Universidad de Chile, de la Dirección del Museo Nacional de Historia Natural, Santiago, por facilitar el acceso a las colecciones osteológicas y a los evaluadores anónimos de Chungara, Revista de Antropología Chilena. 


\section{Anexo 1}

Contexto arqueológico, cultural, económico y tipo de deformación de los cráneos analizados en el presente estudio según

Dembo e Imbelloni (1938) (Tipo 1) y Soto-Heim (1987) (Tipo 2) (CE = circular erecta, CO = circular oblicua, TE = tabular erecta, $\mathrm{TO}=$ tabular oblicua, $\mathrm{ND}=$ sin deformación intencional, DLNI = Deformación leve no intencional, PS = Pseudocircular) (1 = Standen 1995; 2 = Espoueys et al. 1995a; 3 = Espoueys 2003).

Archaeological, cultural and economic context, and type of intentional deformation of the skulls analysed in the present study according to Dembo and Imbelloni (1938) (Tipo 1) and Soto-Heim (1987) (Tipo 2) classifications (CE = Circular erected, $C O=$ Circular oblique, $T E=$ Tabular erected,$T O=$ Tabular oblique, $N D=$ Non deformed, $D L N I=$ Slight non intentional deformation, $P S=$ Pseudo-circular $)(1=$ Standen 1995; 2 = Espoueys et al. 1995a; 3 = Espoueys 2003).

\begin{tabular}{|c|c|c|c|c|c|c|c|c|}
\hline Período & Cronología & Sitio & Cráneo & Grupo Cultural & Economía & Ref. & Tipo 1 & Tipo 2 \\
\hline \multirow[t]{7}{*}{ Arcaico } & 5.000-4.000 a.p. & Pl.Miller-8 & 3496 & Chinchorro & Pesca & 1 & ND & DLNI \\
\hline & & Pl.Miller-8 & 3624 & Chinchorro & Pesca & 1 & ND & ND \\
\hline & & Pl.Miller-8 & 3625 & Chinchorro & Pesca & 1 & ND & DLNI \\
\hline & & Pl.Miller-8 & 3628 & Chinchorro & Pesca & 1 & $\mathrm{CO}$ & $\mathrm{CO}$ \\
\hline & & Pl.Miller-8 & 3634 & Chinchorro & Pesca & 1 & $\mathrm{TE}$ & $\mathrm{TE}$ \\
\hline & & Pl.Miller-8 & 3683 & Chinchorro & Pesca & 1 & ND & $\mathrm{ND}$ \\
\hline & & Pl.Miller-8 & 3736 & Chinchorro & Pesca & 1 & ND & $\mathrm{ND}$ \\
\hline \multirow[t]{7}{*}{ Medio } & $600-1.000$ & Azapa-1 & 3471 & Cabuza & Agrícola & 2 & $\mathrm{CE}$ & $\mathrm{CE}$ \\
\hline & & Azapa-3 & 3452 & Cabuza & Agrícola & 2 & $\mathrm{CO}$ & $\mathrm{CO}$ \\
\hline & & Azapa-3 & 3486 & Maitas & Agrícola & 2 & ND & ND \\
\hline & & Azapa-3 & 3571 & Cabuza & Agrícola & 2 & $\mathrm{CO}$ & $\mathrm{CO}$ \\
\hline & $800-1.000$ & Azapa-3 & $5 \mathrm{~B}$ & Cabuza & Agrícola & 2 & $\mathrm{TE}$ & $\mathrm{TE}$ \\
\hline & & Azapa-103 & 3535 & Tiwanaku & Agrícola & 3 & TE & TE \\
\hline & & Azapa-103 & 3564 & Cabuza & Agrícola & 3 & TO & TO \\
\hline \multirow[t]{13}{*}{$\begin{array}{l}\text { Intermedio } \\
\text { Tardío }\end{array}$} & $1.250-1.460$ & Azapa-8 & T39X & $\begin{array}{l}\text { Gentilar- } \\
\text { San Miguel }\end{array}$ & Agrícola & 3 & $\mathrm{CE}$ & $\mathrm{CE}$ \\
\hline & $1.250-1.460$ & Azapa-8 & 3433 & San Miguel & Agrícola & 3 & $\mathrm{CE}$ & $\mathrm{CE}$ \\
\hline & $1.250-1.460$ & Azapa-8 & 3434 & San Miguel & Agrícola & 3 & $\mathrm{CE}$ & $\mathrm{CE}$ \\
\hline & $1.250-1.460$ & Azapa-8 & 3440 & Gentilar & Agrícola & 3 & $\mathrm{CO}$ & $\mathrm{CE}$ \\
\hline & $1.250-1.460$ & Azapa-8 & 3441 & Gentilar & Agrícola & 3 & $\mathrm{CO}$ & $\mathrm{CO}$ \\
\hline & $1.250-1.460$ & Azapa-8 & 3442 & Gentilar & Agrícola & 3 & $\mathrm{TE}$ & TE PS \\
\hline & $1.250-1.460$ & Azapa-8 & 3565 & Gentilar & Agrícola & 3 & $\mathrm{CO}$ & $\mathrm{CO}$ \\
\hline & $1.250-1.460$ & Azapa-8 & 3592 & San Miguel & Agrícola & 3 & $\mathrm{TE}$ & TE PS \\
\hline & $1.250-1.460$ & Azapa-8 & 11 & $\begin{array}{l}\text { Gentilar- } \\
\text { San Miguel }\end{array}$ & Agrícola & 3 & $\mathrm{TE}$ & TE PS \\
\hline & $1.250-1.460$ & Azapa-79 & 3555 & San Miguel (?) & Agrícola & 3 & $\mathrm{CO}$ & $\mathrm{CO}$ \\
\hline & $1.250-1.460$ & Azapa-79 & 3493 & San Miguel (?) & Agrícola & 3 & $\mathrm{CO}$ & $\mathrm{CO}$ \\
\hline & $1.000-1.460$ & Pica-8 & 3525 & $\begin{array}{l}\text { Pica- } \\
\text { San Miguel }\end{array}$ & Agrícola & 3 & $\mathrm{CO}$ & $\mathrm{CO}$ \\
\hline & $1.000-1.460$ & Pl.Miller-3 & 3488 & $\begin{array}{l}\text { Gentilar- } \\
\text { San Miguel }\end{array}$ & Pesca & 3 & ND & DLNI \\
\hline \multirow[t]{2}{*}{ Tardío } & $1.460-1.580$ d.C. & Lluta-12 & 3516 & $\begin{array}{l}\text { San Miguel } \\
\text { Tardío }\end{array}$ & Agrícola & 3 & $\mathrm{CE}$ & $\mathrm{CE}$ \\
\hline & $1.460-1.580$ d.C. & Lluta-13 & $3523 \mathrm{D}$ & Inca & Agrícola & 3 & TE & TE PS \\
\hline
\end{tabular}




\section{Referencias Citadas}

Allison, M.J., E. Gerszten, J. Munizaga, C.M. Santoro y

G. Focacci

1981 La práctica de la deformación craneana entre los pueblos andinos precolombinos. Chungara 7:238-260.

Allison, M.J., G. Focacci, E. Gerszten, C.M. Santoro y

J. Munizaga

1982 Estudio radiográfico y demográfico de morbilidad y mortalidad de pueblos precolombinos del Perú y Chile. Chungara 8:265-273.

Anton, S.

1989 Intentional cranial vault deformation and induced changes of the cranial base and face. American Journal of Physical Anthropology 79:253-267.

Anton, S.C., C.R. Jaslow y S.M. Swartz

1992 Sutural complexity in artificially deformed human (Homo sapiens) crania. Journal of Morphology 214:321-332.

Arriaza, B.

1993 Seronegative spondyloarthropathies and diffuse idiopatic skeletal hyperostosis in ancient Northern Chile. American Journal of Physical Anthropology 91:263-278.

2003 Cultura Chinchorro: las Momias más Antiguas del Mundo. Editorial Universitaria, Santiago.

Arriaza, B., V.G. Standen, R. Beckett, G. Conlogue, A. Inzulza y G. Madden

2002 Radiological studies of six Chinchorro statuette mummies. En Mummies in a New Millennium: Proceedings of the 4th World Congress on Mummy Studies, editado por N.Y. Lynnerup, C. Andreasen y J. Berglund, pp. 29-33. Greenland National Museum and Archives and Danish Polar Center, Copenhagen.

Aufderheide, A.C. y M.J. Allison

1994 Bioanthropology of spontaneously mummified bodies of a Late Phase Chinchorro site (Morro 1-6) in northern Chile. Ponencia presentada en la $59^{\underline{a}}$ Reunión Anual de la Sociedad Americana de Arqueología, California.

Aufderheide, A.C., S. Aturaliya y G. Focacci 2002 Enfermedades pulmonares de una muestra de población del cementerio AZ-75, Valle de Azapa, norte de Chile. Chungara 34:253-263.

Aufderheide, A., I. Muñoz y B. Arriaza

1993 Seven Chinchorro mummies and the prehistory of Northern Chile. American Journal of Physical Anthropology 91:189-201.

Bass, W.M.

1987 Human Osteology: A Laboratory and Field Manual ( $3^{\text {rd }}$ edition). Special Publication $N^{o} 2$ of the Missouri Archaeological Society. Columbia, Missouri: Missouri Archaeological Society, Inc.

Benett, K.A.

1965 The etiology and genetics of wormian bones. American Journal of Physical Anthropology 23:255-260.

Bird, J.

1943 Excavations in northern Chile. Anthropological Papers of the American Museum of Natural History of New York 38:171-318.

Blom, D., B. Hallgrimsson, L. Keng, M. Lozada y J. Buikstra 1998 Tiwanaku state colonization: Bioarchaeological implications for migration in the Moquegua Valley, Peru. Word Archaeology 30:238-261.
Bookstein, F.

1989 Principal warps: thin-plate spline and the decomposition of deformations. IEEE Transactions in Pattern Analysis and Machine Intelligence 11:567-585.

1991 Morphometric Tools for Landmark Data: Geometry and Biology. Cambridge University Press, Cambridge.

Buikstra, J.E. y D.H. Ubelaker

1994 Standards for data collection from human skeletal remains. Research Series № 44. Arkansas Archaeological Survey, Fayetteville.

Chacama, J.

2004 El Horizonte Medio en los valles occidentales del Norte de Chile (ca. 500-1.200 d.C.). Chungara, Revista de Antropología Chilena, Volumen Especial: 227-233.

Cheverud, J.M. y J.E. Midkiff

1992 Effects of fronto-occipital cranial reshaping on mandibular form. American Journal of Physical Anthropology 87:167-171.

Cheverud, J.M., L.A.P Kohn, L.W. Konigsberg y S.R. Leigh 1992 Effects of fronto-occipital artificial cranial vault modification on the cranial base and face. American Journal of Physical Anthropology 88:323-345.

Cocilovo, J.A.

1975 Estudio de dos factores que influencian la morfología craneana en una población andina: el sexo y la deformación artificial. Revista del Instituto de Antropología (Tucumán) 2:197-212.

1978 Estudio de dos factores que influyen en la morfología craneana en una colección patagónica: el sexo y la deformación artificial. Archivos de Anatomia e Antropologia (Rio de Janeiro) 3:113-141.

1994 Biología de la Población Prehistórica de Pisagua. Continuidad y Cambio Biocultural en el Norte de Chile. Tesis Doctoral, Facultad de Ciencias Exactas, Físicas y Naturales, Universidad Nacional de Córdoba, Argentina.

Cocilovo, J.A., F. Rothhammer, S. Quevedo y E. Llop

1982 Microevolución en poblaciones prehistóricas del área andina. III. La población del Morro de Arica. Craneometría. Revista UNRC (Río Cuarto) 2:91-111.

Dauelsberg, P.

1972 La cerámica de Arica y su situación cronológica. Chungara 1-2:17-24.

Dembo, A. y J. Imbelloni

1938 Deformaciones intencionales del cuerpo humano de carácter étnico. Humanior (Buenos Aires), Sección A, Tomo 3:1-348.

El-Najjar, M. y G.L. Dawson

1977 The effect of artificial cranial deformation on the incidence of wormian bones in the lamdoidal suture. American Journal of Physical Anthropology 46:155-160.

Enlow, D. y M. Hans

1998 Crecimiento Facial. Editorial McGraw-Hill Interamericana, México.

Espoueys, O.

2003 Continuidad y cambio en Pisagua: aproximación al carácter cultural de la deformación craneana intencional en Pisagua; métodos y técnicas. Manuscrito en posesión del autor. 
Espoueys, O., M. Uribe, A. Román y A. Deza

1995a Nuevos fechados por termoluminiscencia para la cerámica del período Medio del Valle de Azapa (Primera Parte). Actas del XIII Congreso Nacional de Arqueología Chilena Vol. 1:31-53. Hombre y Desierto 9, Antofagasta.

Espoueys, O., V. Schiappacasse, J. Berenguer y M. Uribe 1995b En torno al surgimiento de la Cultura Arica. Actas del XIII Congreso Nacional de Arqueología Chilena. Vol. 1:171-185. Hombre y Desierto 9, Antofagasta.

Gerszten, P.

1993 An investigation into the practice of cranial deformation among the pre-Columbian peoples of northern Chile. International Journal of Osteoarchaeology 3:87-98.

Gerszten, P. y E. Gerszten

1995 Intentional cranial deformation: a disappearing form of self-mutilation. Neurosurgery 37:374-382.

Gerszten, P., E. Gerszten y M.J. Allison

1998 Diseases of the skull in Pre-Columbian South American mummies. Neurosurgery 42:1145-1152.

González, F., J.C. Salinas y G. Manríquez

2004 Variación de la forma facial post-tratamiento ortopédico-quirúrgico: Análisis de telerradiografías craneofaciales mediante morfometría geométrica. Ponencia presentada en XVII Annual Meeting International Association for Dental Research Sección Chile, Concepción.

Hunt, D.R. y L.M. Hopper

1996 Non-Invasive investigations of human mummified remains by radiographic techniques. En Man in the Ice: vol.3, Human Mummies: a Global Survey of their Status and the Techniques of Conservation, editado por K. Spindler. Editorial Springer, Viena.

Imbelloni, J.

1925 Deformaciones intencionales del cráneo en Sud América. Revista del Museo de La Plata 28:329-407.

1932 Sobre un ejemplar mimético de deformación craneana: el cráneo 3876 de la Isla de Tilcara (Jujuy, Argentina). Anales del Museo Nacional de Historia Natural 37:193-207. Buenos Aires.

Kendall, D.G.

1984 Shape manifolds, procrustean metrics and complex projective spaces. Bulletin of London Mathematical Society 16:81-121.

Llagostera, A.

1989 Caza y pesca marítima (9.000 a 1.000 a.C.). En Culturas de Chile. Prehistoria. Desde sus Orígenes hasta los Albores de la Conquista, editado por J. Hidalgo, V. Schiappacasse, H. Niemeyer, C. Aldunate e I. Solimano, pp. 57 79. Editorial Andrés Bello, Santiago.

Loy A., L. Mariani, M. Bertelletti y L. Tunesi

1998 Visualizing allometry: geometric morphometrics in the study of shape changes in the early stages of the two-banded sea bream, Diplodus vulgaris (Perciformes, Sparidae). Journal of Morphology 237:137-146.

Lozada, M.C. y J.E. Buikstra

2002 El Señorío Chiribaya en la Costa Sur del Perú. Instituto de Estudios Peruanos, Lima.

Manríquez, G., S. Quevedo y O. Espoueys

2002 Contribución de la morfometría geométrica al estudio de la deformación craneana intencional en poblaciones prehispánicas de Chile. Ponencia presentada en XLV Reunión Anual de la Sociedad de Biología de Chile, Puyehue.
Manríquez, G., F. Sánchez y R. Smith

2003 Morfometría geométrica y reconstrucción tridimensional de la variación de la forma calvaria en poblaciones del sur y extremo austral de Chile. Ponencia presentada en XLVI Reunión Anual de la Sociedad de Biología de Chile, Puyehue.

Manríquez, G., F. González, J.C. Salinas y O. Espoueys

2004 Análisis de morfometría geométrica de la variación de la forma cráneo-maxilar mediante reconstrucción 2D y 3D. Ponencia presentada en XVII Reunión Anual International Association for Dental Research Sección Chile, Concepción.

Manríquez, G. y E. Llop

2004 Bioantropología de las poblaciones del extremo austral. En Poblaciones Chilenas: Cuatro Décadas de Investigaciones Bioantropológicas, editado por F. Rothhammer y E. Llop, pp. 87-104. Editorial Universitaria, Santiago.

McNeill, R.W. y G.N. Newton

1965 Cranial base morphology in association with intentional cranial vault deformation. American Journal of Physical Anthropology 23:241-254.

Munizaga, J.

1964 Deformación cefálica intencional (análisis de algunas poblaciones precolombinas en el norte de Chile). Antropología (Santiago) 2:5-17.

1973 Síntesis de la antropología física del Norte Chico. Actas del VI Congreso de Arqueología Chilena. Vol. 1:345351. Boletín de Prehistoria, Número Especial, Santiago.

1975 Introducción a la Antropología Física (Curso dictado en el Departamento de Ciencias Antropológicas y Arqueológicas de la Universidad de Chile, compilado por E. Aspillaga). Santiago.

Muñoz, I.

1989 El período Formativo en el norte grande (1.000 a.C. a 500 d.C.). En Culturas de Chile. Prehistoria. Desde sus Orígenes hasta los Albores de la Conquista, editado por J. Hidalgo, V. Schiappacasse, H. Niemeyer, C. Aldunate e I. Solimano, pp. 107-128. Editorial Andrés Bello, Santiago. 2004 Estrategias de Organización Prehispánica en Azapa: el Impacto de la Agricultura en un Valle del Desierto Costero del Pacífico. Editorial Universidad de Tarapacá, Arica.

O'Higgins, $\mathrm{P}$.

2000 The study of morphological variation in the hominid fossil record: biology, landmarks and geometry. Journal of Anatomy 197:103-120.

O'Loughlin, V.D.

1996 Comparative endocranial vascular changes due to craniosynostosis and artificial cranial deformation. American Journal of Physical Anthropology 101:369-385.

Ossenberg, N.

1970 The influence of artificial cranial deformation on discontinuous morphological traits. American Journal of Physical Anthropology 33:357-372.

Özbek, M.

2001 Cranial deformation in a subadult sample from Degirmentepe Chalcolithic, Turkey. American Journal of Physical Anthropology 115:238-244.

Rhode, M.P.

2002 Cranial deformation and measurement stability among prehistoric south central Andean populations. American Journal of Physical Anthropology [Suppl] 33:130. 
Rohlf, F.J.

2000 NTSYS-pc. Numerical Taxonomy and Multivariate Analysis System, v. 2.1a. Exeter Software (programa computacional).

2001 TPSdig, v. 1.31. NY: State University at Stony Brook (programa computacional).

2003a TPSrelwarp, v. 1.35. NY: State University at Stony Brook (programa computacional).

2003b TPSreg v. 1.27. NY: State University at Stony Brook (programa computacional).

Rohlf, F.J. y L. Marcus

1993 A revolution in morphometrics. Trends in Ecology and Evolution 8:129-132.

Romero, A., C.M. Santoro, D. Valenzuela, J. Chacama,

E. Rosello y L. Piacenza

2004 Túmulos, ideología y paisaje de la Fase Alto Ramírez del Valle de Azapa. Chungara, Revista de Antropología Chilena, Volumen Especial: 261-272.

Rothhammer, F., S. Quevedo, J.A. Cocilovo, G. Focacci y

E. Llop

1982 Microevolución en poblaciones prehistóricas del área andina. II. Variación craneométrica cronológica en los valles de Arica. Chungara 8:275-289.

Rothhammer, F., J.A. Cocilovo, S. Quevedo y E. Llop 1983 Afinidad biológica de las poblaciones prehistóricas del litoral ariqueño con grupos poblacionales costeros peruanos y altiplánicos. Chungara 13:99-108.

Rothhammer, F., S. Quevedo, J.A. Cocilovo y E. Llop 1984 Microevolution in prehistoric Andean populations: II. Chronologic nonmetrical cranial variation in northern Chile. American Journal of Physical Anthropology 66:157-162.

1989 Orígenes y microevolución de la población chilena. En Culturas de Chile. Prehistoria. Desde sus Orígenes hasta los Albores de la Conquista, editado por J. Hidalgo, V. Schiappacasse, H. Niemeyer, C. Aldunate e I. Solimano, pp. 403-413. Editorial Andrés Bello, Santiago.

Rothhammer, F., C.M. Santoro y M. Moraga

2002 Craniofacial chronological microdifferentiation of human prehistoric populations of the Azapa valley, northern Chile. Revista Chilena de Historia Natural 75:259-265.

Rothhammer, F. y C. Silva

1990 Craniometrical variation among South American prehistoric populations: climatic, altitudinal, chronological, and geographic contributions. American Journal of Physical Anthropology 82:9-17.

Salinas, J.C., F. González, O. Espoueys y G. Manríquez

2004 Comparación de análisis cefalométrico y de morfometría geométrica en cráneos prehispánicos de Chile. Ponencia presentada en XVII Annual Meeting International Association for Dental Research Sección Chile, Concepción.

Santoro, C.M., A. Romero, V.G. Standen y A. Torres

2004 Continuidad y cambio en las comunidades locales, períodos Intermedio Tardío y Tardío, valles occidentales del área Centro Sur Andina. Chungara, Revista de Antropología Chilena, Volumen Especial: 235-247.

Schiappaccasse, V., V. Castro y H. Niemeyer

1989 Los desarrollos regionales en el norte grande (1.000 a 1.400 d.C.). En Culturas de Chile. Prehistoria. Desde sus Orígenes hasta los Albores de la Conquista, editado por J. Hidalgo, V. Schiappacasse, H. Niemeyer, C. Aldunate e I. Solimano, pp. 181-220. Editorial Andrés Bello, Santiago.
Spielman, R.

1972 Anthropometric and Genetic Differences Among Yanomana Villages. Doctoral Dissertation, University of Michigan, Massachusetts.

Soto-Heim, P.

1987 Evolución de deformaciones intencionales, tocados y prácticas funerarias en la prehistoria de Arica, Chile. Chungara 19:129-213.

Standen, V.G.

1995 La colección Chinchorro de Playa Miller 8. Informe Proyecto Fondecyt № 1930202.

2003 Bienes funerarios del Cementerio Chinchorro Morro 1, descripción, análisis e interpretación. Chungara, Revista de Antropología Chilena 35:175-208.

Standen, V.G., M.J. Allison y B. Arriaza

1984 Patologías óseas de la población Morro-1, asociada al complejo Chinchorro: Norte de Chile. Chungara $13: 175-185$

Standen, V.G. y B. Arriaza

$2000 \mathrm{La}$ treponematosis (Yaws) en las poblaciones prehispánicas del desierto de Atacama (Norte de Chile). Chungara 32:185-192.

Standen, V.G., C. Santoro y B. Arriaza

2004 Síntesis y propuestas para el Período Arcaico en la costa del extremo Norte de Chile. Chungara, Revista de Antropología Chilena, Volumen Especial: 201-212.

Stewart, T.D.

1943 Skeletal remains from Paracas, Peru. American Journal of Physical Anthropology 1:47-63.

Stewart, T.D. y M.T. Newman

1963 Anthropometry of South American indian skeletal remains. En Handbook of South American Indians Vol. 6 Physical anthropology, linguistics and cultural geography of South American indians, editado por J.H. Steward, pp. 19-42. Cooper Square Publishers, New York.

Sutter, R.C. y L. Mertz

2004 Nonmetric cranial crait variation and prehistoric biocultural change in the Azapa Valley, Chile. American Journal of Physical Anthropology 123:130-145.

Thompson, D.

1980 [1917] Sobre el Crecimiento y la Forma. Traducido por J.M. Beas. Editorial Hermann Blume, Madrid.

Trinkaus, E.

1982 Artificial cranial deformation in the Shanidar 1 and 5 Neandertals. Current Anthropology 23:198-199.

Varela, H. y J.A. Cocilovo

2002 Genetic drift and gene flow in a prehistoric population of the Azapa Valley and coast, Chile. American Journal of Physical Anthropology 118:259-267.

Weiss, P.

1961 Osteología Cultural. Prácticas cefálicas, Segunda Parte. Anales de la Facultad de Medicina (Lima) 44:133-277.

Varela, H. y J.A. Cocilovo y S. Quevedo

1995 La edad como factor de variación intramuestral en la población prehistórica de San Pedro de Atacama, Chile. Chungara 27:125-134.

Weiss, $\mathrm{P}$.

1962 Tipología de las deformaciones cefálicas de los antiguos peruanos, según la osteología cultural. Revista del Museo Nacional (Lima) 31:15-42.

White, C.D.

1996 Sutural effects of fronto-occipital cranial modification. American Journal of Physical Anthropology 100:397-410.

Uhle, $\mathrm{M}$.

1919 La arqueología de Arica y Tacna. Boletín de la Sociedad Ecuatoriana de Estudios Históricos Americanos 3:1-49. 


\section{Notas}

1 Con el fin de facilitar tanto la comprensión de la composición cultural de la muestra como la comparación de nuestros resultados con los obtenidos por otros autores, además de la tipología de Dembo e Imbelloni (1938) que es la usada en el presente trabajo, en el Anexo 1 hemos agregado la tipología usada por Munizaga (1975) y Soto-Heim (1987). Estos autores, buscando mejorar la comprensión de las variantes observadas en el grupo de las deformaciones anulares (circulares), y basándose en Dembo e Imbelloni (1938), proponen una quinta categoría de clasificación, la pseudocircular, que permite discriminar formas que presentan características de tabulares pero que, en la práctica, fueron realizadas aplicando vendajes, una particular variante de los aparatos circulares. La categoría pseudocircular permite una mejor aproximación a las técnicas de deformación, resueltas por Imbelloni como tabulares erectas en el caso de las deformaciones tabulares realizadas con uso de superficies rígidas (tablillas o cuna), pero no abordadas a fondo en el caso de las variaciones generadas por el uso de técnicas basadas en vendajes. Por último, aunque Dembo e Imbelloni (1938) incorporan el grado de intensidad deformadora entre los caracteres con que definen su tipología, cuando esta es muy leve la forma resultante tiende a aproximarse más a la de los cráneos no deformados que a los de su propia tipología deformadora. Por ello, SotoHeim (1987) y Lozada y Buikstra (2002) clasifican las deformaciones leves no intencionales en una categoría aparte.

2 Cabe destacar que debido a las características intrínsecas del material proveniente de excavaciones, el presente estudio es de carácter exploratorio, ya que no están representados los períodos Temprano e Intermedio Temprano.

3 Los programas de morfometría geométrica fueron implementados por F.J. Rohlf (State University of New York, Stony Brook, NY) y obtenidos desde el sitio web http:// life.bio.sunysb.edu/morph. Los análisis discriminante y de la varianza se llevaron a cabo con las rutinas del programa STATISTICA. El análisis de agrupamiento por UPGMA y la proyección bivariada de las matrices de los valores de relative warp se efectuó mediante el programa NTSYS-pc (Rohlf 2000).

4 Una posible explicación de este hecho es que el grupo de cráneos circular horizontales es casi exclusivo de sitios costeros del período Intermedio Tardío, poco representados en la abundante Colección del Museo Arqueológico San Miguel de Azapa. 